'Ilu. Revista de Ciencias de las Religiones

ISSN: $1135-4712$

http://dx.doi.org/10.5209/ILUR.53840

\title{
Minervium vs. Minerva Capta: due facce della stessa medaglia?
}

\author{
Tiziano Cinaglia ${ }^{1}$
}

Recibido: 9 de agosto de 2015 / Aceptado: 12 de noviembre de 2015

Resumen. La vulgata moderna ha sempre identificato il Minervium varroniano con i parva delubra menzionati da Ovidio relativamente al culto di Minerva Capta; si tratta, in effetti, dello stesso tempio, individuabile sull'altura del Celio. Sulla base di una analisi etimologica, linguistica ed infine storica, vengono esaminati i due diversi nomi, sottolineando le implicazioni religiose e cultuali che se ne ricavano: il Minervium, contestualizzabile soltanto grazie all'arcaica lista dei sacella degli Argei; Minerva Capta, invece, epiclesi fondamentale per la comprensione del culto, connesso ai riti d'iniziazione post-puberali. Da questo esame si evidenzia come entrambe le espressioni si colleghino a fasi arcaiche della religione romana e, pertanto, viene dimostrata anche l'arcaicità del culto stesso di Minerva, sia per quanto riguarda il Celio, ma più in generale per la storia della comunità romana.

Palabras clave: Argei; Capio, -е̌re; Evocatio; Riti d'iniziazione; Minerva Capta; Minervium.

\section{[en] Minervium vs. Minerva Capta: two sides of the same coin?}

\begin{abstract}
Modern scholars have always associated the Minervium of Varro with the parva delubra mentioned by Ovid, which is related to the cult of Minerva Capta: in fact, these two different names identify the same temple, located on the Cealius hill. Therefore, this work examines the etymological, linguistic and historical context of both names of Minerva's shrine, by pointing out any religious and cultual implications concerning such expressions: the Minervium, connected to the archaic list of sacella Argeorum; the epithet Capta, whose meaning is fundamental to the comprehension of a cult connected with post-pubertal transition rites. In conclusion, both expressions must be linked to the archaic period of the Roman religion and so demonstrate the antiquity of Minerva's cult, as it regards the Celius hill but also the history of the whole Roman community.
\end{abstract}

Keywords: Argei; Capio, -е̌re; Evocatio; Minerva Capta; Minervium; Transitionals rites.

Sumario. 1. Introduzione. 2. Minervium. 3. "(Il tempio) di Minerva". 4. Minerva Capta. 5. Una dea evocata? 6. Una dea 'sposata'? 7. Una dea 'accolta'; o piuttosto, una dea 'che prende'. 8. Conclusioni. 9. Bibliografia.

Cómo citar: Cinaglia, T. (2016), Minervium vs. Minerva Capta: due facce della stessa medaglia?, en 'Ilu. Revista de Ciencias de las Religiones 21, 51-78. 


\section{Introduzione}

In un noto passo del De Vita Caesarum, Svetonio ci racconta come Caligola abbia condotto sua figlia Drusilla in giro per la città in modo da farle visitare tutti i templi delle dee dell'Urbe, terminando quello che dobbiamo immaginare come un lunghissimo tour religioso per la Roma primo-imperiale esattamente al tempio di Minerva - non sappiamo quale - dove l'imperatore, da ultimo, affidò sua figlia alla dea, affinché quest'ultima fungesse da tutrice nella crescita e nell'educazione della bambina ${ }^{2}$. Come già ricordato, non è noto in quale santuario si sia svolta questa scena, né tanto meno quali templi siano stati effettivamente oggetto delle attenzioni dell'imperatore e di sua figlia; ma probabilmente, considerata la predilezione che Caligola mostra nei riguardi di Minerva, è probabile che abbiano visitato, tra tutti i templi romani, anche e soprattutto quelli della dea: ovvero, nel dettaglio, il celeberrimo Minervium varroniano sul Celio ${ }^{3}$, tempio noto anche come delubrum Minervae Captae dai versi ovidiani ${ }^{4}$, ma anche l'importantissimo santuario dell'Aventino ${ }^{5}$, il meno celebre tempio di Minerva Medica dell'Esquilino ${ }^{6}$ ed infine, last but not least, la cella consacrata alla dea nel tempio capitolino. Ora, per dare un nome ai suddetti templi, la letteratura moderna usa convenzionalmente gli scarsi dati, letterari e non, di cui oggigiorno disponiamo; ma, andando al dunque, la domanda che dobbiamo pórci è la seguente: i Romani, concretamente, come si riferivano ai loro templi? Ossia, quali termini, quali espressioni usavano, nella loro quotidianità, per definire questi luoghi di culto, più o meno importanti e più o meno presenti nelle dinamiche della vita cittadina? Purtroppo, nella maggior parte dei casi, non siamo assolutamente informati a riguardo: il più delle volte, in effetti, dobbiamo 'accontentarci' del nome istituzionale, tramandatoci dall'antiquaria, nome generalmente costituito dall'epiclesi della divinità - come ad esempio nel caso della Minerva dell'Esquilino ${ }^{7}$; o addirittura limitarci ad una generica indicazione della divinità, senza ulteriori dettagli

2 Svet., Cal., XXV, 4: Infantem autem, Iuliam Drusillam appellatam, per omnium dearum templa circumferens Minervae gremio imposuit alendamque et instituendam commendavit.

3 Varr., Ling. Lat., V, 47: [Huic iunctae] Carinae et inter eas quem locum Caeriolensem appellatum apparet, quod primae regionis quartum sacrarium scriptum sic est: Caeriolensis: quarticeps circa Minervium qua in Caelium montem itur: in tabernola est.

$4 \quad$ Il termine delubrum in relazione al tempio del Celio è tratto dal celebre passo ovidiano dei Fasti (III, 809-848) dedicato alla festa delle Quinquatrus del 19 marzo, che al verso 837 recita: Parva licet videas Captae delubra Minervae. Da Ovidio sappiamo che il santuario si trovava lungo una strada nel punto in cui questa iniziava a scendere dal Celio (Ov., Fast., III, 835-836: Caelius ex alto qua mons descendit in aequum, / Hic ubi non plana est sed prope plana via), mentre grazie a Varrone sappiamo che il versante era quello rivolto verso l'Esquilino ( $c f r$. n. 3); pertanto, il santuario va collocato nella parte settentrionale della collina, sul Caeliolus, presso l'odierna chiesa dei SS. Quattro Coronati (Colini 1944, 39; Platner - Ashby 1965, 344; Jordan - Hülsen 1871-1907, I.3, 226; Schürmann 1985, 10; Girard 1989, 164; Coarelli 1996, 255; idem 1998, 210).

5 Conosciuto grazie a diverse fonti (Fest., 446L; Liv., XXVII, 37, 7; Ov., Fast., VI, 725-728; RGDA, 19.2) ed alla Forma Urbis (FUR, tav. XXIII, 22 b). Per una bibliografia essenziale sul santuario aventinese: Valentini Zucchetti 1940, 140, 181 e 244; Platner - Ashby 1965, 342; Jordan - Hülsen 1871-1907, I.3, 159s.; Cassatella Vendittelli 1985, 442ss.; Schürmann 1985, 9,63ss.; Richardson 1992, 254s.; Ziolkowski 1992, 109ss.; Vendittelli 1996, 254.

$6 \quad$ Identificato sulla base di due iscrizioni con dedica alla dea $\left(C I L \mathrm{I}^{2} 460=C I L\right.$ VI 30980; CIL VI 10133) ed in base ai dati del deposito votivo portato alla luce sul finire del XIX secolo in Via Carlo Botta. Per una bibliografia essenziale sul santuario dell'Esquilino: Platner - Ashby 1965, 344s.; Jordan - Hülsen 1871-1907, I.3, 352s.; Gatti lo Guzzo 1978; Schürmann 1985, 11; Richardson 1992, 256; Carlucci 1996, 255s.

7 CIL VI 10133: $D \cdot M / C n \cdot$ Vergilius / Epaphroditus / magister · odatarius / a Minerva / Medica. Iscrizione funeraria, proveniente da Via Merulana, dall'area del santuario e databile alla fine dell'età repubblicana. 
né informazioni - come è per la Minerva dell'Aventino ${ }^{8}$. Ma nel caso del tempio del Celio, invece, dobbiamo ritenerci fortunati, poiché siamo verosimilmente di fronte sia al nome ufficiale del culto, quello celeberrimo di Minerva Capta $^{9}$, ma anche all'espressione con cui era più comunemente conosciuto nella vita di tutti i giorni, al termine quotidianamente usato dagli abitanti di Roma, ovvero il sostantivo Minervium ${ }^{10}$.

Ora, mentre l'epiteto Capta può contare su una letteratura imponente, con molteplici tentativi di interpretazione, alcuni dei quali credibili, altri invece decisamente discutibili - un tema sul quale tornerò più diffusamente in seguito -, il sostantivo Minervium ed il contesto in cui viene citato, al contrario, non hanno mai destato in particolar modo l'interesse degli studiosi moderni. L'epiteto Capta è stato, a più riprese, usato come fulcro nei vari tentativi esegetici che di volta in volta prefiguravano una Minerva già arcaicamente presente a Roma, o che, al contrario, la escludevano dall'originario pantheon romano facendone una dea straniera; sempre e comunque, pertanto, elemento centrale della discussione sul culto e sull'origine della dea. Il termine Minervium, invece, puntualmente passato sotto silenzio, inosservato, quasi scontato; e, a mio modesto parere, oserei dire abbastanza inspiegabilmente ${ }^{11}$.

\section{Minervium}

Vorrei pertanto iniziare la mia analisi proprio da tale argomento, poiché ritengo che questo termine meriti in realtà un esame più dettagliato ed approfondito; e non solo perché sinora trascurato, ma soprattutto in quanto latore di importanti, e forse risolutive, informazioni sul tempio del Celio. Come precedentemente accennato, è il passo di Varrone ad informarci di questa denominazione più 'colloquiale', se così possiamo definirla, per il tempio della Minerva ceriolense; ma non si tratta di una espressione coniata dall'erudito reatino, né tanto meno di una semplice banalizzazione del nome del santuario in epoca tardo-repubblicana: infatti, il contesto in cui il Minervium viene citato è il celebre brano che elenca i sacraria Argeorum della prima Regione Suburana. Come è noto, i passi varroniani in cui vengono descritti i vari sacella delle quattro Regiones sono comunemente interpretati come trascrizioni dirette di diversi frammenti dei libri pontificales ${ }^{12}$, dove la disposizione dei sacrari

\footnotetext{
FUR, tav. XXIII, 22 b: ... MINERBAE.

Cfr. n. 4.
}

10 Di avviso opposto sono Torelli e la Donati, i quali sostengono invece che si debba riconoscere nel termine $\mathrm{Mi}$ nervium il nome ufficiale del culto (Torelli 1984, 52; Donati - Stefanetti 2006, 38).

11 Gli unici, e solo in epoca recentissima, che hanno in parte e molto rapidamente sottolineato l'importanza di questo termine sono la Storchi Marino (Storchi Marino 1979, 353), la Marroni (Marroni 2010, 267 n. 285) ed infine Pedroni (Pedroni 2011, 40). In precedenza, solo Torelli (Torelli 1984, 57) aveva debitamente posto in evidenza l'importanza del contesto in cui il Minervium viene citato, ovvero la lista degli Argei.

12 O libri augurum, ma in ogni caso un testo ex sacris Argeorum (Saglio 1969, 404; Palmer 1970, 84; Harmon 1978, 1447; Coarelli 1993a, 120; Storchi Marino 1995, 267 e 276s.; Scheid 1999, 162). Secondo Jordan (Jordan - Hülsen 1871-1907, II, 239s.) questo testo era un "Ritualurkunde", proveniente dalla raccolta delle prescrizioni rituali conservate negli archivi pontificali; ed allo stesso modo anche Wissowa e Rohde considerano la lista come un documento degli archivi pontificali (Wissowa 1904, 211: "Akt des Staatskultes"; Rohde 1936, 60). Palmer e Poe parlano di un "handbook" che si sarebbe caratterizzato per delle frasi corte e senza legami sintattici (Palmer 1970, 84 n. 2; Poe 1978, 149); solo Collart che, come gli altri studiosi, descrive l'elenco come "un chapitre des livres pontificaux", si interroga su quale testo si legga oggi: quello dei pontefici o piuttosto un testo rivisitato da Varrone, o addirittura modificato dai copisti successivi? (Collart 1954, 175). Scheid, da ultimo, non 
ed il loro ordine nella processione erano rigorosamente indicati per uno scrupolo evidentemente rituale. Secondo la letteratura moderna, la fonte cui attingeva Varrone era una redazione di età medio-repubblicana di un testo evidentemente $\operatorname{arcaico}^{13}$; conseguentemente, possiamo considerare come certa l'esistenza del Minervium già nel III sec. a.C., dal momento che, all'interno dell'elenco dei loca sacra degli Argei, il terminus post quem per una collocazione cronologica della fonte varroniana, in altre parole il tempio più recente da quest'ultima menzionato, è quello di Quirino, che fu dedicato agli inizi del III sec. a.C. dal console L. Papirio Cursore ${ }^{14}$.

Abbiamo quindi evidenziato il nesso tra l'arcaico rituale degli Argei ed il Minervium $^{15}$; ma reputo che questo sostantivo possa ancora darci ulteriori dettagli in merito alle vicende del santuario del Celio, a prescindere dalla complessa disamina sulla datazione del testo ex sacris Argeorum. Maggiori informazioni, infatti, si possono ricavare da una semplice analisi linguistica del termine, ossia dall'esame del suo originario valore lessicale: si tratta, è evidente, di un aggettivo, derivato dal nome proprio della dea, il cui significato è 'proprio di Minerva', 'pertinente a Minerva'; a

ritiene invece verisimile l'ipotesi di un testo specifico dei Sacra Argeorum, perché non vi sono prove dell'esistenza dei cosiddetti "grandi libri sacerdotali", e ritiene di conseguenza che, verosimilmente nel I sec. a.C., "les antiquaires se sont inspirés directement des commentarii accessibles et des décrets sacerdotaux conservés par les archives du sénat. De toute façon, cela ne change rien à la nature des textes en question, qui étaient, selon toute évidence, des résumés de célébrations et des textes de décisions", risalenti al III sec. a.C. (Scheid 1999, 166). Per una bibliografia aggiornata sul tema $c f r$. A. Quaglia, Argei, nemici e voodoo dolls, c.d.s.

13 De Sanctis 1953, IV.2.1, 146; Palmer 1970, 85; Harmon 1978, 1452; Torelli 1984, 53; Coarelli 1993a, 120; Storchi Marino 1995, 277 e 286; Scheid 1999, 164; Phillips 2006, 940. La datazione al III sec. a.C. del testo sacro degli Argei al quale attinge Varrone è realizzata, dalla maggior parte degli studiosi, proprio sulla base della menzione del Minervium, utilizzato come terminus post quem e la cui fondazione è erroneamente fatta risalire al 241 a.C., anno della conquista di Falerii. Coarelli, invece, sostiene che, come terminus post quem, sia da utilizzarsi la dedicatio della aedes Salutis, tempio costruito nel 302 a.C. (Coarelli 1993a, 124); mentre Torelli, come Phillips, pur non prendendo una posizione definitiva, afferma però che il Minervium non sia databile al 241 a.C. proprio perché inserito in questo arcaico rituale degli Argei. Scheid, infine, sostiene che se "la forme non achaique du texte parait corroborer cette datation", ovvero al III sec. a.C. per la fonte varroniana, d'altro canto "il est évident que les rites et la tradition peuvent etre plus anciens" (Scheid 1999, 164).

14 Il tempio, costruito in seguito ad un voto di L. Papirio Cursore nel 325 a.C., dopo una vittoria sui Sanniti, fu però dedicato soltanto 30 anni più tardi, dal figlio omonimo, nel 293 a.C. (Liv., X, 46, 7).

15 Ora, considerando la formularità ed il conservatorismo religioso, tipico di qualsiasi religione ed in particolar modo di quella romana, se da un lato dobbiamo ritenere che i sacraria siano rimasti inalterati per ordine e numero nella successione sin dall'epoca arcaica, come mera ipotesi di lavoro è forse ipotizzabile che, per la processione sacra degli Argei, siano stati usati anche gli stessi riferimenti topografici, quando questi erano già presenti: ed infatti, credo che valga la pena sottolineare come, per la quasi totalità dei luoghi citati nell'elenco varroniano, si possa far risalire l'origine del culto ad un'epoca molto alta, spesso arcaica. Ė questo il caso della maggior parte dei luci menzionati, come quello del Facutalis (Stara-Tedde 1905, 199ss.; Coarelli 1993a, 123; Buzzetti 1996a, 193) e quello dell'Esquilinus (Stara-Tedde 1905, 201s.; Coarelli 1993a, 123; Buzzetti 1996b, 193); ma è soprattutto il caso di tutte le divinità che si incontrano lungo il percorso: a partire da Iuno Lucina, il cui culto è connesso alla mitica ara di Tito Tazio (Varr., Ling. Lat., V, 75 - Stara-Tedde 1905, 204ss.; Ziolkowski 1992, 67ss.; Coarelli 1993a, 124; Giannelli 1996b, 122s.); per proseguire poi con il tempio di Quirinus, dedicato nel 293 a.C. (Liv., X, 46, 7), ma sicuramente sul luogo di un culto arcaico (Ziolkowski 1992, 139ss.; Coarelli 1999a, 185ss.); successivamente incontriamo il tempio della dea Salus, di origine antichissima e probabilmente in origine praticato in un sacello all'aperto (Ziolkowski 1992, 144ss.; Coarelli 1993a, 124; Coarelli 1999b, 229s.); anche il culto di Semus Sancus Dius Fidius è collegato, dall'antiquaria, al passato mito-storico di Roma, fondato secondo le differenti tradizioni o da Tito Tazio (Tert., ad Nat., II, 9, 28) o da Tarquinio il Superbo (Dion. Hal., Quaest. Rom., IX, 60, 8) (Coarelli 1999c, 263s.). Inoltre, anche la aedes Romuli, che sia da identificare con un tempio o con la casa del fondatore di Roma, ha evidentemente una origine arcaica (Coarelli 1993b, 241s.); ed infine il tempio dei Penates, che è posto in relazione con la casa di Tullo Ostilio (Varr., in Non, 531, 19; Sol., 1, 25) (Ziolkowski 1992, 128ss.; Palombi 1999, 75ss.). Si tratta, ovviamente, di una mera considerazione, che non pretende in nessun modo di assurgere a valore di indizio dell'arcaicità del tempio ceriolense; quello che però possiamo fino ad ora desumere con certezza, è che la lista degli Argei, ed il Minervium in essa elencato, possono datare a partire degli anni iniziali del III sec. a.C. 
questo proposito, gli esempi della lingua latina non sono numerosi, ma sono comunque incontrovertibili: conosciamo, infatti, i versus Minervii ${ }^{16}$, un'espressione traducibile come 'canti, inni di Minerva', che insieme agli Axamenta formavano i carmina Saliaria; Tito Livio e Velleio Patercolo, inoltre, facendo riferimento con ogni probabilità alla medesima colonia calabrese, utilizzano rispettivamente le espressioni di oppidum Minervium e di Scolacium Minervia, ad indicare una 'città di Minerva'17. Abbiamo inoltre notizia di una legio Minervia ${ }^{18}$, della prima età imperiale; ed infine Arnobio utilizza questo aggettivo sia col significato di 'pertinente a Minerva', ma anche come aggettivo sostantivato, riferendosi in questo caso ad un tempio ateniese ${ }^{19}$, essendo ormai totalmente compiuto il sincretismo religioso tra Atena e la dea romana. Sull'impiego di questo aggettivo, ancora in epoca medio-repubblicana, siamo addirittura documentati dal punto di vista epigrafico, poiché conosciamo due iscrizioni che ci testimoniano nuovamente dell'uso dell'aggettivo menervius/-a: una prima iscrizione, incisa su di un'ara proveniente dalla etrusca Veio, riporta in latino l'espressione [M]inerv[i]a, esplicitabile come 'altare di Minerva'20; la seconda iscrizione, infine, scritta in lingua osca e rinvenuta nell'area del santuario di Punta Campanella, reca l'aggettivo menereviius connesso al sostantivo medd [i] ks, personaggi che dovevano svolgere funzioni di magistrati o piuttosto operare come sacerdoti di Minerva ${ }^{21}$.

\section{3. "(Il tempio) di Minerva"}

Che si trattasse originariamente di un aggettivo, pertanto, è indubbio, data la sua presenza ed il suo utilizzo come tale, senza soluzione di continuità, dalla media età repubblicana sino all'epoca imperiale, nelle fonti letterarie come nei documenti epigrafici; un comune aggettivo, dunque, indicante genericamente tutto ciò che è pertinente a Minerva. Altrettanto evidente, però, è che, in un dato momento, non individuabile, della storia romana, questo aggettivo assunse successivamente il valore di nome proprio, per indicare solo ed esclusivamente il tempio della dea sul Celio ${ }^{22}$.

16 Paul. Fest., 3L: Axamenta dicebantur carmina Saliaria, quae a Saliis sacerdotibus componebantur, in universos homines composita. Nam in deos singulos versus ficti a nominibus eorum appellabantur, ut Ianuli, Iunonii, Minervii.

17 Liv., XLV, 16, 5: Aedes deum Penatium in Velia de caelo tacta erat et in oppido Minervio duae portae et muri aliquantum; Vell. Pat., I, 15, 4: et, post annum, (deducta est) Scolacium Minervia. La colonia di cui parlano i due autori è la cittadina di Squillace, dedotta nel 123 a.C. e così chiamata per la presenza di un importante culto di Minerva-Atena.

18 Scriptores Historiae Augustae, II, Spartiani, De vita Adriani, 3, 6: Secunda expeditione Dacica Traianus eum primae legioni Minerviae praeposuit secumque duxit. La legione mantenne il nome che le era stato dato da Domiziano nell'82 d.C.

19 Arn., ad. Nat., IV, 16, 23: Minervium nomen (il nome di 'Minerva'); V, 26, 16: Minervii cives (cittadini di Atene); VI, 6, 9: in Minervio (nell'area del tempio di Atena).

$20 \quad C I L \mathrm{I}^{2} 2629=$ ILLRP 28. L'iscrizione è databile al III secolo a.C. (cfr. Rix 1981, 120; Mezzetti 1997-2000, 190s.).

21 Iscrizione databile alla metà del II sec. a.C.: $m \cdot$ gaaviis $m \cdot l \cdot$ pitakiis $\cdot m / l \cdot$ appuliis $\cdot$ ma $\cdot$ medd [i]ks menereviius / esskazsium · ekuk · upsannum / dedens iusum · prufattens (Prosdocimi 1989, 531s.; Russo 1990, 191ss.).

22 La stessa sorte toccò, paradossalmente, anche ad un secondo aggettivo relativo a Minerva, minervalis, $-e$, che nella sua forma neutra passò a significare, per lo meno dall'epoca tardo-repubblicana, obolo che gli studenti dovevano pagare ai loro precettori nel giorno delle Quinquatrus (Varr., Re Rust., III, 2, 18: Axius: "Merula miinquit - recipe me quaeso discipulum villaticae pastionis. - Ille: "Qui simulac promiseris minerval, incipiam" - inquit [id est cenam]; Hier., in Eph., VI, 4: minervale munus <quod> grammaticus et orator aut in sumptos domesticos, aut in temple stipes, aut in sordida scorta convertit). 
Chi, ormai nel corso dell'ultimo secolo e mezzo, si è occupato in generale del culto di Minerva, e del tempio del Celio più in particolare ${ }^{23}$, non ha mai avvertito la necessità di tradurre questo nome proprio, per utilizzare una terminologia grammaticale, risultando evidentemente superflua una trasposizione in una lingua moderna per la palese ed immediata comprensibilità del termine stesso $^{24}$; ma volendo renderlo in italiano, potremmo forse pensare ad una espressione come 'Minervio', oppure più banalmente, esplicitandolo, ad una perifrasi quale 'Tempio di Minerva': infatti il termine Minervium deve verosimilmente essere il risultato della semplificazione di una originaria espressione 'templum minervium', sintagma che, una volta omesso per brevità il sostantivo templum, si è ridotto all'impiego del solo aggettivo ormai sostantivato $^{25}$. La validità di tale interpretazione è corroborata dall'esistenza di un Dianium ${ }^{26}$, santuario connesso alla domus di Servio Tullio e già scomparso in epoca augustea, un nome che, come afferma la Storchi Marino, "ha il chiaro senso di locus Dianae sacratus" 27 , ovvero di 'templum dianium': si tratta, a mio avviso, di due casi assimilabili, entrambi riconducibili ad espressioni successivamente 'apocopate' per questioni di economicità e semplificazione linguistica, nelle quali il sostantivo templum risultava ormai quasi ridondante. Sulla base di quanto detto ritengo pertanto che il termine Minervium possa interpretarsi come una espressione dall'origine più 'colloquiale', nata dalla prassi quotidiana, in relazione al tempio ceriolense; ma non per questo dobbiamo però considerare questa espressione meno valida o meno ufficiale, ché avremo modo di vedere come abbia verosimilmente tutti i connotati di arcaicità ${ }^{28}$.

Ora, proprio nella trasposizione del termine Minervium come 'Tempio di Minerva' risiede, invero, il presupposto per portare alla luce un dato fondamentale sulle origini del santuario ceriolense: infatti, dobbiamo sottolineare che, al momento in cui scriveva Varrone, a Roma esistevano con certezza almeno altri due templi di Minerva ${ }^{29}$, ovvero i precedentemente citati templi dell'Aventino, esistente almeno dalla fine

23 L'analisi della figura di Minerva prende le mosse dalle fondamentali opere di Preller (Preller 1858, 258ss.), di Gilbert (Gilbert 1883-1890, II, 26ss. e 233ss.) e di Wissowa (Wissowa 1897, 2982ss.); ma sin dai primi studi, il termine Minervium sarà lasciato nella sua originaria forma latina. Non ho qui dimenticato gli altrettanto fondamentali testi di H. Usener (Italische Mythen, in "Rheinisches Museum für Philologie", XXX 1875, 221ss.) e di W. Warde Fowler (Roman Festivals of the Period of the Republic, London 1899, 57ss.); ma questi autori, nell'indagine da loro effettuata sulle caratteristiche della dea, non presero in considerazione né l'epiteto Capta, né tanto meno il termine Minervium.

24 Gli unici che parlano di "Tempel der Minerva" sono Walde e Hofmann (Walde - Hofmann 1938, 90).

25 Ma si può anche pensare all'ipotetico impiego di un originario termine quale delubrum, piuttosto che al sostantivo templum, in associazione all'aggettivo minervium, così come ci suggeriscono i versi ovidiani.

26 Il Dianium, ovvero il tempio di Diana, è noto da un passo di Livio $(\mathrm{I}, 48,6)$ e da un'iscrizione di provenienza urbana (CIL VI, 33922): il santuario, scomparso da poco in età augustea, era collocato all'angolo tra il Vicus Cuprius ed il Vicus Urbius e doveva essere connesso alla domus di Servio Tullio.

27 Storchi Marino 1979, 353.

28 Simili espressioni sembrano potersi rilevare piuttosto diffusamente nella fase arcaica; sono noti, in effetti, vari aggettivi neutri sostantivati, aventi la funzione di individuare uno specifico edificio sacro: è il caso, ad esempio, del Lupercal, dell'Apollinar o del Volcanal. Va inoltre sottolineato che una costruzione pressoché identica da un punto di vista morfologico a quella del nome Minervium, poiché formata dal medesimo suffisso produttivo -io-, è quella che si rileva nei termini greci Heraion, Artemision, etc., un dato che può forse evidenziare una comune tendenza nel mondo greco-latino, una sorta di specializzazione di questo suffisso per la genesi di tali sostantivi nel periodo arcaico.

29 In realtà, all'epoca di Varrone doveva esistere anche un ulteriore santuario dedicato a Minerva: infatti, i Cataloghi Regionari costantiniani menzionano una aedes Minervae per la Regio I (165, $207 \mathrm{VZ} \mathrm{I),} \mathrm{che} \mathrm{è} \mathrm{probabilmente}$ da localizzarsi presso Porta Latina, sulla base di un rinvenimento archeologico effettuato nel XVIII secolo, e da identificarsi, inoltre, con uno degli arcaici santuari collocati al I miglio (Colonna 1991, 209ss., con bibliografia sul tema). 
del III secolo a.C..$^{30}$, e dell'Esquilino, noto archeologicamente già a partire dal IV secolo a.C. ${ }^{31}$ Pertanto, senza tenere in considerazione il tempio del Campidoglio, mai indicato dalle fonti come tempio di Minerva ${ }^{32}$, dobbiamo però domandarci perché, in età tardo-repubblicana, uno dei tre templi della dea, e soltanto questo, fosse definito come 'Tempio di Minerva': non si correva il rischio di creare confusione, di essere fraintesi, impiegando una generica espressione quale è quella di 'Tempio di Minerva' per riferirsi invece ad un unico e determinato santuario, esistendo contemporaneamente per lo meno altre due aedes della dea? Ovviamente la risposta è negativa; e la motivazione è, ancor più categoricamente, immediatamente individuabile: infatti, la comunità romana, adesso come allora, certamente impiegava nel linguaggio quotidiano espressioni sintetiche, più 'adatte' alla lingua parlata, per riferirsi a realtà determinate $\mathrm{e}$ ben individuabili, ma senza ambiguità alcuna, perché uniche $\mathrm{e}$ comunemente note ${ }^{33}$; in altri termini, ritengo che la causa della nominalizzazione di questo aggettivo sia da ricercare necessariamente nell'unicità e nella condizione di 'non-confondibilità' del santuario del Celio, ovvero nella sua antichità e nell'importanza che esso aveva per la primitiva comunità romana. Ovviamente non possiamo sapere se, ancora alla metà del I sec. a.C., al momento in cui scriveva Varrone, il santuario ceriolense, per la communis opinio, fosse ancora generalmente considerato come il 'Tempio di Minerva', ossia come il santuario più importante della dea nel panorama religioso romano: quello che l'autore reatino usa, infatti, è ormai diventato, in età tardo-repubblicana, un semplice sostantivo, e non possiamo essere certi che significasse, che volesse ancora evidenziare le stigmate della preminenza del tempio del Celio sugli altri santuari urbani dedicati alla dea ${ }^{34}$; quello di cui siamo però certi è che la fossilizzazione dell'aggettivo come nome proprio del tempio non può che risalire ad un'epoca in cui quest'ultimo costituiva l'unico culto destinato alla dea, quindi verosimilmente il culto principale di Minerva durante tutta l'età repubbli-

30 Al santuario aventinese si fa riferimento per la prima volta nel periodo della seconda guerra punica, per un evento del 207 a.C.: si tratta di un prodigium, o meglio di vari prodigia, che avevano sconvolto Roma e che, per ordine dei pontefici, furono espiati per mezzo di un inno composto da Livio Andronico e cantato da 27 vergini. La notizia liviana del prodigium e della successiva espiazione (Liv., XXVII, 37, 1-10: ... Decrevere item pontifices, ut virgines ter novena per urbem euntes carmen canerent. Id cum in Iovis Statoris aede discederent conditum ab Livio poeta carmen, tacta de caelo aedis in Aventino Iunonis Reginae ...) ci viene completata da Festo, grazie al quale sappiamo del 'coinvolgimento' del tempio aventinese in questa vicenda (446L: Itaque cum Livius Andronicus bello Punico secundo scripsisset carmen ... publice adtributa est ei in Aventino aedis Minervae, in qua liceret scribis histrionibusque consistere ac dona ponere).

31 Cfr. n. 6.

32 Infatti al tempio del Campidoglio le fonti letterarie facevano comunemente riferimento con il termine Capitolium (cfr. in merito Tagliamonte 1996a, 226s.) oppure con l'espressione Iuppiter Optimus Maximus (cfr. Tagliamonte 1996b e De Angeli 1996).

33 Per poter comprendere questa condizione, mutatis mutandis, basterebbe un rapido esame di una qualsiasi città contemporanea; ma per fare un parallelo con una realtà certamente nota a tutti, ritengo sia lecito il confronto con la situazione della Roma attuale: oggigiorno, infatti, si utilizza comunemente l'espressione 'San Pietro' per indicare ovviamente la Basilica di San Pietro in Vaticano; ma a Roma la Basilica Vaticana non è certamente l'unica chiesa consacrata all'apostolo Pietro, dal momento che abbiamo anche le chiese di San Pietro in Carcere, San Pietro in Vincoli, San Pietro in Montorio e S. Pietro in Borgo. Se pertanto possiamo, senza timore di essere fraintesi, impiegare per motivi di praticità una espressione abbreviata e sintetica, ma col chiaro intento di riferirci solo ed esclusivamente alla Basilica Vaticana, è perché quest'ultima costituisce senza dubbio il luogo di culto per eccellenza dedicato a San Pietro, il luogo di culto più importante e più antico di tutta la città.

34 Molto probabilmente, in epoca tardo-repubblicana, il culto più importante di Minerva era ormai diventato quello dell'Aventino, sia per la monumentalità del santuario (documentata dalla Forma Urbis - cfr. n. 5), che certamente doveva invece mancare al parvum delubrum del Celio, sia per la centralità che esso dovette assumere nei festeggiamenti delle varie corporazioni artigianali cittadine, in seguito alla concessione a scribae ed histriones nel 207 a.C. di celebrarvi la propria festa (cfr. n. 30). 
cana: infatti, come abbiamo visto in precedenza, il tempio di Minerva Medica dell'Esquilino è databile al IV secolo, e pertanto il Minervium deve necessariamente datare ad un'epoca anteriore. Dobbiamo però considerare che il IV secolo, in questo nostro ragionamento, costituisce semplicemente un terminus ante quem, ed il V secolo non può quindi rappresentare la datazione ultima del nostro santuario: infatti, se accettiamo, sulla base di quanto appena detto, che il tempio del Celio fosse precedente agli altri due santuari dedicati a Minerva, il più antico dei quali risale al IV sec. a.C., non possiamo però evidentemente dedurre, sulla base di questa semplice considerazione, di quanto fosse più antico il Minervium, ossia a che epoca dovesse effettivamente risalire. Pertanto, non è da escludere che questo santuario esistesse in una fase ancora più alta, che fosse già presente nel periodo $\operatorname{arcaico}^{35}$; ed anzi, proseguendo con questa analisi, vedremo come quest'ultima ipotesi sia più che una mera possibilità.

Ora, infatti, non ci rimane che investigare l'altro lato della medaglia, quello più noto e dibattuto, ovvero l'etimologia dell'epiteto Capta. Il passo al cui interno è menzionata la ormai 'famigerata' Minerva Capta non necessita di particolari introduzioni: si tratta della descrizione ovidiana, nei Fasti, della festa delle Quinquatrus del 19 marzo $^{36}$; in altre parole, il testo più ampio, circostanziato e ricco di particolari su culti e miti concernenti la dea. In questa sede, però, non è ovviamente possibile analizzarlo in dettaglio e ci limiteremo quindi al solo esame dell'epiclesi Minerva Capta, ovvero a quello che ritengo essere il 'nome ufficiale' del santuario del Celio: gli studiosi moderni, infatti, non hanno mai avuto alcun dubbio sull'identità tra Minervium e Minerva Capta, entrambi i termini impiegati dalle fonti antiche per indicare un luogo di culto della dea sul colle Celio. Abbiamo un'unica voce che discorda dal coro in merito a questa identificazione, ossia quella della Storchi Marino ${ }^{37}$ : la studiosa, infatti, in un lavoro non più recentissimo, aveva messo in discussione la credibilità di questa duplice denominazione per il medesimo santuario; ed il dubbio nasce, aggiungerei a buon diritto, da una osservazione molto puntuale ed attenta, che sottolinea quella che invero costituisce una incongruenza sostanziale tra l'esegesi generalmente proposta per l'origine di Minerva Capta e quella che, invece, è attribuibile al Minervium: in effetti, come abbiamo avuto modo di esaminare in precedenza, il termine Minervium denota una sicura antichità del culto tributato alla dea del Celio, termine connesso ai rituali degli Argei ed assimilabile a quello del Dianium liviano precedentemente citato, anch'esso sicuramente arcaico $^{38}$; ma il tempio della Minerva Capta ovidiana è, al contrario, generalmente datato dalla vulgata moderna alla metà del III sec. a.C., come avremo immediatamente modo di esaminare, proprio sulla base del significato con cui comunemente si interpreta questa epiclesi, con particolare riferimento al participio passato capta.

Avremmo, di conseguenza, una aporia sostanziale tra le due definizioni del santuario: la prima, quella di Minervium, che ci riconduce ad una realtà arcaica, e la seconda, quella di una Minerva capta, che è invece cronologicamente collocabile solo dopo la metà del III sec. a.C.; pertanto la Storchi Marino giunge a sostenere

35 I sostenitori dell'arcaicità del Minervium sono la Storchi Marino (Storchi Marino 1979, 353), Torelli (Torelli 1984, 52s.), Coarelli (Coarelli 1998, 212ss.), Ley (Ley 2000, 212) e Pedroni (Pedroni 2011, 40).

36 Ov., Fast., III, 809-848.

37 Storchi Marino 1979, 353.

38 Anche questo tempio sembrerebbe presentare tutti i connotati di arcaicità, come per il santuario del Celio, dal momento che era connesso, secondo la tradizione, con la domus di Servio Tullio (cfr. F. Coarelli, s. v. Dianium, LTUR 2, Roma 1995, 15s.). 
come non sia del tutto convincente l'assimilazione tra il Minervium ed il tempio di Minerva Capta e che, data la presenza del Minervium in "un documento antico e sacro" quale è quello della lista degli Argei, con questo termine si "potrebbe forse alludere ad un (altro) sacellum consacrato a Minerva" ${ }^{39}$, sempre da collocarsi sul Celio. La conclusione cui giunge la Storchi Marino è certamente da confutare, e dobbiamo continuare ad attribuire i due diversi nomi al medesimo santuario del Celiolo, non avendo né testimonianze archeologiche, né altre fonti che ci documentino di un secondo tempio di Minerva sul colle del Celio; però rimane, evidentemente, da risolvere l'incongruenza rilevata da questa puntuale osservazione.

\section{Minerva Capta}

Ma quali sono, in concreto, le varie letture che la letteratura dà dell'epiclesi Minerva Capta? Le ipotesi interpretative sono state, da più di un secolo e mezzo a questa parte, numerose e spesso molto difformi: Preller, unico, alla metà dell'Ottocento, ipotizzò che l'epiteto Capta derivasse da caput, come d'altronde suggerisce lo stesso Ovidio $^{40}$, in quanto Minerva dea della mente, che è sede dell'intelligenza, ovvero dell'ingenium $^{41}$; ma questa teoria ebbe scarsa fortuna nella letteratura scientifica successiva, essendo oltretutto priva di un qualsiasi fondamento scientifico ed etimologico. Gilbert invece, qualche decennio più tardi, suppose che questa epiclesi derivasse alla dea dalla sua relazione con il Capitolium $^{42}$, sottolineandone l'antichità del culto; ma lo stesso Preller, quasi contestualmente a Gilbert, in una successiva revisione della sua fondamentale opera Römische Mythologie, propose una nuova interpretazione dell'aggettivo capta $^{43}$, una teoria poi divenuta a tutti gli effetti 'verità assoluta' per quasi un secolo e che tuttora continua a godere di notevole credito, ossia quella che traduce, sulla scorta dell'ipotesi ovidiana ${ }^{44}$, l'epiteto capta con il sostantivo 'prigioniera', affermando che "der Name kann wohl nur die 'Gefangene' bedeuten" "45: infatti, negli ultimi versi dedicati alla descrizione delle Quinquatrus, il poeta dei Fasti dispiega il suo ventaglio eziologico e la terza etimologia proposta, tra le quattro elencate, è per l'appunto quella secondo la quale il termine Capta sarebbe stato attribuito a Minerva dopo la presa di Falerii del 241 a.C. e quindi introdotta a Roma come "prigioniera" da Q. Lutatius Catulus Cerco: questa spiegazione è stata spesso, ed in maniera del tutto acritica, accolta in toto dalla vulgata moderna, per la quale la soluzione ovidiana, prefigurante una origine falisca e conseguentemente una 'cattività' della dea, rappresentava la sola interpretazione possibile dell'epiteto in questione ${ }^{46}$, senza mai giungere, peraltro, a considerare le eventuali implicazioni

\footnotetext{
Cfr. n. 37.

Ov., Fast., III, 839-840: Capitale vocamus / Ingenium sollers: ingeniosa dea est.

Preller 1858, 260; Preller - Jordan 1881-1883, I, 292.

Gilbert 1883-1890, II, 85.

Preller - Jordan 1881-1883, I, 292 n. 2.

Ov., Fast., III, 843-844: An quia perdomitis ad nos captiva Faliscis / Venit? Et hoc signo littera prisca docet.

Cfr. n. 43.

La letteratura a riguardo è praticamente sterminata ed è pertanto obiettivamente impossibile citare tutti coloro che, di volta in volta, anche con semplici contributi o riferimenti puntuali, abbiano sostenuto questa tesi; cercherò, quindi, di menzionare per lo meno le opere più importanti. Di questo avviso, infatti, è la maggioranza degli studiosi moderni, a partire da Wissowa, a più riprese (Wissowa 1897, 2984s.; idem 1899, 1554; idem 1902, 203) e da Aust (Aust 1899, 143), dagli autori del Thesaurus (TLL, III, s. v. capio, 335), da Altheim (Altheim 1932, 1777;
} 
di ordine religioso e cultuale che questo appellativo avrebbe comportato per il culto tributato alla dea.

Gli studiosi moderni hanno preso frequentemente le mosse da questi versi, nell'accingersi ad esaminare la figura di Minerva, avendo l'aggettivo capta sempre calamitato l'attenzione di qualsiasi tentativo di analisi sulla dea; ma le motivazioni addotte a supporto di una presunta 'prigionia' di Minerva, al di là del passo ovidiano dei Fasti, non sono né numerose né, onestamente, degne di credibilità: come prima immediata obiezione, infatti, dobbiamo evidenziare che lo stesso Ovidio, tutt'altro che certo della sua esegesi, prima di intraprendere tale esame etimologico afferma che nominis in dubio causa est (v. 839); al termine dell'invocazione alla dea torna poi a ribadire il concetto, concludendo che si augura l'appoggio di Minerva a quacumque trahis ratione vocabula (v. 847). È evidente, quindi, che il poeta dei Fasti non fosse affatto sicuro relativamente alle sue proposte para-etimologiche e che, di conseguenza, come ha sottolineato Torelli, queste titubanze del poeta sono da interpretarsi come "rivelatrici dell'incapacità dell' antiquaria romana di risalire nel tempo e di comprendere l'origine del culto" 47 , indizi, pertanto, dell'incertezza che, agli inizi dell'età imperiale, regnava sull' origine del tempio del Celio ${ }^{48}$. Secondariamente, non è che una mera considerazione, priva pertanto di validità documentaria e probatoria, il fatto che la moglie di Ovidio fosse originaria di Falerii Novi e che, di conseguenza, il poeta dovesse essere ben informato sui fatti e quindi conoscere con certezza la provenienza del culto ceriolense ${ }^{49}$ : per quanto proveniente da Falerii Novi, anche la moglie non doveva indubbiamente avere informazioni di prima mano, essendo ormai trascorsi due secoli e mezzo dalla presa della città al momento della stesura

idem 1951-53, I, 195s.), da Enking (Enking 1945, 111) e da Brelich (Brelich 1949, 110 n. 65), per continuare poi con De Sanctis (De Sanctis 1953, IV.2.1, 146), Gagé (Gagé 1955, 242), Bömer (Bömer 1957-58, II, 201), Latte (Latte 1960, 164), con la Terrosi Zanco (Terrosi Zanco 1961, 192), con Radke (Radke 1965, 218), Dumézil (Dumézil 1966, 414s.), Bollini (Bollini 1969, 351), Fougères (Fougères 1969, 1928s.), con Girard, anch'egli a più riprese (Girard 1970, 469; idem 1981, 206; idem 1989, 166); ed ancora con Bruun (Bruun 1972, 120), Frazer (Frazer 1973, 151) e con la Storchi Marino (Storchi Marino 1979, 353). Poi, in epoca più recente, proseguiamo con Bloch (Bloch 1980, 351), Schilling (Schilling 1981, 112; idem 2003, 161 n. 242), Scullard (Scullard 1981, 94), Canciani (Canciani 1984, 1075), con la Porte (Porte 1985, 64s.), con Martin 1987 (Martin 1987, 52), Sabbatucci (Sabbatucci 1988, 134), Le Bonniec (Le Bonniec 1990, 227 n. 185), Miller (Miller 1992, 27), Montanari (Montanari 1993, 271), Peruzzi (Peruzzi 1997, 72) ed infine, ormai nel nostro secolo, con la Gustafsson (Gustafsson 2000, 58), Graf (Graf 2001, 132), Orlin (Orlin 2002, 15 n. 13) e La Bua (La Bua 2010, 55). Dumézil è addirittura giunto a sostenere che "ce nom cultuel (scil. Capta) ... il ne fait allusion, on le voit, qu'à un événement historique et n'enseigne rien sur sa nature" (Dumézil 1966, 301), negando inequivocabilmente qualsiasi valore religioso e cultuale all'epiteto. Non tutti gli autori che sostengono una provenienza falisca, e quindi una cattività di Minerva, sono però concordi sulla datazione di questa evocatio: Hild, infatti, sostiene che Minerva sia stata evocata nel 394 a.C., quando Furio Camillo ottenne, senza combattere, la resa della città di Falerii (Hild 1969c, 802).

47 Torelli 1984, 52. Dello stesso avviso anche Ziolkowski (Ziolkowski 1992, 113s.) e la Donati (Donati - Stefanetti 2006, 38).

48 Dobbiamo sottolineare che alle etimologie, ed a tutto il passo ovidiano sulle Quinquatrus, sia soprattutto da attribuire un valore letterario, poetico, con una particolare finalità narrativa: infatti, come è stato a più riprese evidenziato, in tutto il mese di marzo dei Fasti ovidiani l'intento principale del poeta è quello di "oscuramento e neutralizzazione" del dio guerriero Marte, per rendere più 'elegiaca' la materia letteraria; e questo obiettivo viene raggiunto attraverso l'esaltazione, per contrasto, delle altre divinità, tra le quali Minerva, e delle loro caratteristiche 'civili' e 'politiche'. Pertanto Ovidio, in quest'ottica, "tende a 'cancellare' la figura di Marte dal mese a lui tradizionalmente consacrato, favorendo una divinità legata alla pace e all'arte” (La Bua 2010, 48); in questo caso rivolgendosi a Minerva, esaltata come dea delle arti, dei mestieri e dell'ingenium (sul tema della 'demilitarizzazione' di Marte e sul ruolo di Minerva dea 'civile', con il contemporaneo esaltamento dell'aspetto elegiaco-amoroso $c f r$. anche Hinds 1992, 89ss.; Miller 1992, 22ss.; Barchiesi 1994, 12ss. e 112ss.; Newlands 1996, 330ss.; Merli 2000, 121ss.).

49 Graf 2001, p. 132. Sappiamo della provenienza della moglie di Ovidio grazie alle sue stesse parole, quando ne fa menzione in un poema degli Amores (III, 13, 1): Cum mihi pomiferis coniunx foret orta Faliscis. 
dei $\mathrm{Fasti}^{50}$; e vale forse la pena ricordare che Ovidio, per la composizione della sua opera, poteva inoltre disporre ed attingere ai testi della tradizione annalistica e letteraria romana, sicuramente meglio informata e più attendibile a riguardo ${ }^{51}$.

Per quanto concerne, invece, l'antica iscrizione menzionata dal poeta, la littera prisca (v. 844), non abbiamo ovviamente alcuna ulteriore indicazione dalle fonti a riguardo, né tanto meno testimonianze archeologiche dirette ${ }^{52}$; pertanto, questi versi, prestandosi l'espressione ad una duplice interpretazione, sono stati utilizzati sia come conferma dell'attestazione di un culto arcaico a Falerii poi trapiantato a Roma, traducendo la frase con 'l'antica iscrizione lo dimostra' - essendo in lettere falische $-^{53}$, ma anche semplicemente come la probabile prova della presenza di un antico simulacro della dea all'interno del santuario, intendendo invece il termine signo usato da Ovidio con il significato di 'statua' e l'espressione prisca littera col valore di 'lettere con caratteri arcaici' ${ }^{54}$. Che vi fosse un'iscrizione dedicatoria con caratteristiche arcaiche e che, inoltre, questa si riferisse ad una statua posta all'interno del santuario, è del tutto verosimile; anzi, lo stesso Ovidio ce lo propone come un dato certo e non abbiamo motivo di dubitarlo. Ma questa testimonianza non può essere usata in alcun modo come prova certa dell'origine falisca della Minerva Capta, data l'ambiguità della frase e soprattutto la scarsa verosimiglianza della menzione, nella dedica dell'iscrizione, di una cattura della dea; in effetti, già ad un sommario sguardo delle iscrizioni dedicatorie oggi conosciute, appare evidente come l'interpretazione che vede nel signum la prova della 'cattività' di Minerva sia poco credibile: nelle dediche ex-voto, perché tale doveva essere la dedica presente nel santuario, vediamo generalmente la presenza del destinatario del dono, ovvero la divinità (in dativo); del - o dei - dedicante (in nominativo); e della circostanza della dedica o del voto stesso, espressioni quasi sempre formulari (domum dat, votum solvit, libens merito, etc.). Ma mai riferimenti a 'divinità catturate' ${ }^{55}$.

50 Può forse sembrare superfluo, o addirittura pedantesco, sottolineare il fatto che l'epiteto Capta fu ovviamente attribuito a Minerva dai Romani, quali che siano le ipotesi a riguardo; e cosa potessero pensare i Falisci, oltretutto a distanza di secoli, non ha certo valore documentario, mentre è certamente più importante notare l'incertezza che regnava nella tradizione annalistica romana, questa sì di decisiva importanza ai fini del nostro ragionamento.

51 È lo stesso Ovidio che, in due diversi passi dei Fasti, menziona rapidamente quelle che dovettero essere le sue fonti principali: il primo passo è all'interno del prologo (I, 7), dove afferma che sacra recognosces annalibus eruta priscis; e la medesima espressione userà anche nel secondo passo in questione (IV, 11). Non si tratta, evidentemente, di una dichiarazione di intenti, né tanto meno di veridicità di uno storiografo; ma, per quanto ci riguarda, solo la dimostrazione che il poeta potesse disporre di materiale di prima mano.

52 Conosciamo un'unica iscrizione, molto probabilmente connessa al culto di Minerva, proveniente da quest'area del Celio: si tratta di una iscrizione rinvenuta negli Horti Theophilis, databile al II secolo a.C. e dedicata dal collegio dei cornicines (CIL VI 524): Miner[vae] / donum [dat] / conlegi[um cor] / nicin [um] (Colini 1944, 39; Jordan - Hülsen 1871-1907, I.3, 227; Coarelli 1998, 212). Ma non si tratta, evidentemente, dell'iscrizione cui fa cenno Ovidio. Per quanto riguarda, invece, l'interpretazione dell'espressione littera prisca, sul finire dell'Ottocento Gilbert aveva proposto, oltre alla lettura comunemente accettata di 'antica iscrizione', anche quella di un possibile riferimento all'antico testo dei libri prontificii (Gilbert 1883-1890, II, $31 \mathrm{n} .1$ ).

53 Bömer 1957-58, I, 175: "Eben dies bezeugt die alte Inschrift."; Frazer 1976, 183: "This very fact is attested by an ancient inscription.”; Schilling 2003, 96: "Ce rensegneiment nous est donné par 1'antique inscription de la statue."

54 Torelli 1984, 53; Girard 1989, 165; Ziolkowski 1992, 114; Coarelli 1998, 212; Donati - Stefanetti 2006, 38; Pedroni 2011, 48. A favore dell'interpretazione del termine signum con la parola 'statua', cfr. CIL $\mathrm{I}^{2}, 1508=\mathrm{X}$, $6508=I L L R P 71 / C I L \mathrm{I}^{2}, 42=\mathrm{XIV}, 4270=I L L R P 82 / C I L \mathrm{I}^{2}, 626=\mathrm{VI}, 331=I L L R P 122 / C I L \mathrm{I}^{2}, 1503=\mathrm{X}$, $5961=I L L R P 135 / C I L \mathrm{I}^{2}, 2247=I L L R P 289$.

55 Ora, rimanendo in ambito falisco, possiamo esaminare a mo' d'esempio, a tal proposito, una nota lamina bronzea inscritta, dedicata per l'appunto a Minerva e proveniente dal sito di Falerii Novi, ovvero da Santa Maria di Falleri; infatti, credo che questa iscrizione possa essere esplicativa ed esemplificativa, poiché si tratta di una dedica ufficiale, fatta per volontà del senato e con il coinvolgimento di un magistrato locale, ovvero una situazione probabilmente simile a quella che dobbiamo immaginare per la dedica ovidiana del santuario del Celio: CIL $\mathrm{I}^{2}$ 
Il passo ovidiano, però, se pone dei dubbi sull'origine del termine capta, ci riferisce invece esplicitamente di una dea proveniente da Falerii; ovvero, se da un lato i Romani non conoscevano più con esattezza l'etimologia dell'epiteto, però d'altro canto sapevano evidentemente di una Minerva venuta dal centro falisco: è chiaro, pertanto, che a Roma doveva esistere una tradizione annalistica che raccontava della conquista di Falerii con conseguente evocatio della divinità poliadica; purtroppo non siamo documentati in merito, a causa della lacuna liviana per il III secolo, ma il passo dei Fasti lascia presupporre che effettivamente la Minerva dei Falisci sia stata evocata nel 241 a.C. ${ }^{56}$ Ed è altresì plausibile che, agli inizi dell'età imperiale, il termine capta venisse genericamente interpretato e banalizzato così come racconta Ovidio; ovvero che la tradizione popolare, nell'incertezza sull'etimologia dell'epiteto, ne avesse ormai distorto il significato originario trasformando Minerva in una dea 'prigioniera'. Ma se non abbiamo motivo di credere che il poeta abbia 'creato' tout court una dea proveniente da Falerii, non possiamo però accettare l'etimologia che la vuole 'catturata' come conseguenza di un rifiuto verso la sua evocatio.

\section{Una dea evocata?}

Si è più volte fatto riferimento ad una probabile evocatio della dea; ed è esattamente questo il fulcro della questione, l'elemento dirimente per la nostra analisi: infatti, tutti i sostenitori di una estraneità di Minerva al pantheon romano, o per lo meno di una origine falisca del culto ceriolense, si sono sempre appellati a questo arcaico rituale per spiegare l'arrivo della dea a Roma: Minerva, evocata, letteralmente 'chiamata fuori', si sarebbe rifiutata di abbandonare la città di Falerii e, pertanto, sarebbe stata 'fatta prigioniera' e quindi condotta a Roma. È questa l'interpretazione tradizionalmente proposta per la Minerva Capta del Celio.

$\mathrm{Ma}$ in questa lettura dell'epiclesi Capta, anche tra gli stessi suoi sostenitori a volte traspare una sorta di 'disagio' nel dover ammettere una tale empietà da parte dei Romani verso la dea ${ }^{57}$; inoltre, tale esegesi denota, a mio parere, forse anche

$365=$ CIL XI $3081=\operatorname{ILLRP} 238=$ CIE $8340=$ ILS II, 1, 3124: Menerva sacru(m) / [L]a(rs) Cotena La(rtis) f(ilius) pretod de / zenatuo sententiad vootum / dedet. Cuando datu rected / concaptum (per una bibliografia aggiornata su questa iscrizione cfr. Mezzetti 1997-2000,190). Altrettanto interessante è il confronto con uno dei celebri tituli Mummiani, nel quale possiamo osservare un voto ed una dedica fatti ex manubiis (ILLRP $122=$ $C I L \mathrm{I}^{2}, 626$ = CIL VI, 331): L. Mummi(us) L.f. co(n)s(ul). / Duct(u), / auspicio imperioque / eius Achaia capt(a). Corinto / deleto Romam redieit / triumphans. / Ob hasce / res bene gestas quod / in bello voverat, / hanc aedem et sign(um) / Herculis Victoris / imperator dedicat.

56 La Minerva introdotta in seguito alla presa di Falerii, secondo una credibile ipotesi proposta da Torelli (Torelli 1984, 53), sarebbe da identificarsi nel culto del tempio in Aventino: molti templi aventinesi, in effetti, risalgono per l'appunto al III secolo (come quelli di Consus, di Libertas, di Iuppiter Fulgur, di Luna); inoltre, l'Aventino sembrerebbe avere anche una relazione privilegiata con i culti frutto di evocatio, dal momento che proprio su questo colle furono costruiti i templi di Iuno Regina (392 a.C.), evocata da Veio ad opera di Furio Camillo, e di Vortumnus (264 a.C.), evocato da Marco Fulvio Flacco in seguito alla conquista di Volsinii.

57 Bloch, invero, soffermandosi al termine della sua analisi su Minerva proprio su questo aspetto, conclude la sua esposizione considerando che "l'espressione (scil. 'Capta') è curiosa da parte di un popolo che ha sempre cercato di conciliarsi le grazie delle divinità dei popoli vinti” (Bloch 1980, 351); Colonna, invece, evitando qualsiasi giudizio, afferma però che la tradizione sull'origine falisca della Minerva Capta non doveva essere unanime (anche se in realtà l'unica fonte nota è quella ovidiana - Colonna 1984, 1051), mentre Scheid sostiene che, sebbene "Falerii sembra aver avuto fra i suoi culti pubblici un importante culto di Minerva, non è per niente sicuro che la cappella di Minerva Capta sia in relazione colla presa della città" (Scheid 2008, 85). Definirei infine semplicemente improponibile la teoria di Miller - successivamente accettata anche da La Bua (La Bua 
un po' di superficialità nell'approccio verso quello che sicuramente è un rituale dai connotati arcaici, dato più volte sottolineato dalla letteratura moderna ${ }^{58}$. Invero l'evocatio era un rituale tramite il quale "i Romani letteralmente 'chiamavano fuori' la divinità protettrice della città nemica assediata, pregandola di abbandonare la sua dimora e i suoi protetti e di accettare di trasferirsi a Roma, dove le sarebbero stati consacrati un culto e un tempio"59; era, in altri termini, una invocazione alla divinità tutelare della città assediata, un invito "ad abbandonare i loro adoratori e diventare protettrice dei Romani e della loro città" 60 . E che si trattasse di una preghiera, volta ad una incorporazione pacifica della divinità poliadica della città conquistata, lo sappiamo inoltre da Macrobio ${ }^{61}$, il quale afferma in maniera categorica, quasi a volerci trarre d'inganno, che i Romani nefas aestimarent deos habere captivos; Servio aggiunge, inoltre, che, senza il previo abbandono della divinità, la conquista della città non sarebbe stata possibile $\mathrm{o}$ che, ancor peggio, si sarebbe commesso un sacrilegio $^{62}$. La celebrazione di tale rito ${ }^{63}$ avveniva poco prima che l'esercito sferrasse l'attacco finale alla città assediata, quando la vittoria era ormai sicura ${ }^{64}$; " $i$ Romani infatti, distinguendo attentamente tra abitatori umani e divini del luogo, non potevano prescindere dall'assenso della divinità civica alla conquista: diversamente si sarebbe commessa un'empietà e messa a rischio la pax deorum" ${ }^{65}$. Pertanto, lo scopo di questo rituale era quello di ottenere il sostegno della divinità straniera alla causa di Roma, e quindi indirettamente il permesso da parte del dio a conquistare la città protetta, eliminando così il rischio di commettere un sacrilegio; inoltre, se con l'abbandono della protezione divina la città risultava di fatto 'desacralizzata', il salvataggio dei sacra stranieri risultava invece fondamentale, perché permetteva di "aumentare il 'potere' divino dalla parte dei Romani" ${ }^{66}$, col passaggio della nuova divinità all'interno del pantheon cittadino. Ma in questo rituale dobbiamo vedere

2010,56) - poiché pone sul piano di sentimenti ed emozioni umane quali 'dignità' e 'imbarazzo', categorie evidentemente pertinenti a tutt'altro ambito, ovvero al culto ed al rito: egli infatti sostiene che, essendo a suo dire l'aetion della conquista di Falerii quello corretto, "the capture of Minerva, baldly stated as the reason for this name, would have hardly added to the dignity of the goddess being celebrated throughout Rome at this festival" e che, pertanto, "to avoid any such embarrassment Ovid offers four possible explanations for the title, and then, as it were, gives responsibility for deciding among them ... to Minerva herself." (Miller 1992, 27).

58 Per una bibliografia essenziale sul tema: Wissowa 1907; Basanoff 1947; Dumézil 1966, 414s.; Bruun 1972; Le Gall 1976; Gustafsson 2000; Sacco 2004, 329ss.; Ferri 2006, 205ss.; Ferri 2010a; Ferri 2010b (con ampia bibliografia aggiornata in Ferri 2010a).

59 Ferri 2010a, 33.

60 Le Gall 1976, 522. Da evidenziare a riguardo anche le preghiere ricostruite da Pighi per le evocationes degli dei di Cartagine e di Veio (Pighi 1958, 72ss.).

${ }_{61}$ Macrobio (Sat., III, 9, 2) ci spiega questo rituale con le seguenti parole, credo in nessun modo fraintendibili: Constat enim omnes urbes in alicuius dei esse tutela, moremque Romanorum arcanum et multis ignotum fuisse ut, cum obsiderent urbem hostium eamque iam capi posse confiderent, certo carmine evocarent tutelares deos; quod aut aliter urbem capi posse non crederent, aut etiam si posset, nefas aestimarent deos habere captivos.

62 Servio, allo stesso modo che Macrobio, è molto chiaro per quanto riguarda la finalità dell'evocatio (in Aen., II 351): Excessere quia ante expugnationem evocabantur ab hostibus numina propter vitanda sacrilegia.

63 Le altre fonti su questo rituale sono quelle di Plinio il Vecchio (Nat. Hist., XXVIII 18: Verrius Flaccus auctores ponit quibus credatur in obpugnationibus ante omnia solitum a Romanis sacerdotibus evocari deum cuius in tutela id oppidum esset promittique illi eundem aut ampliorem apud Romanos cultum. Et durat in pontificum disciplina id sacrum, constatque ideo occultatum in cuius dei tutela Roma esset, ne qui hostium simili modo agerent.) e di Festo (268 L: Peregrina sacra appellantur quae aut evocatis dis in oppugnandis urbibus Romam sunt conlata, aut quae ob quasdam religiones per pacem sunt petita, ut ex Phrygia Matris Magnae, ex Graecia Cereris, Epidauro Aesculapi: quae coluntur eorum more, a quibus sunt accepta.)

${ }_{64}$ Macr., Sat., III, 9, 2; Liv., V, 21, 1. Ferri 2010a, 34.

65 Ferri 2010a, 29.

66 Ferri 2010a, 35. 
anche e soprattutto una volontà politica, una prassi volta ad 'indebolire' il centro appena conquistato privandolo della divinità poliadica ma, allo stesso tempo, finalizzata ad una più rapida integrazione, prima religiosa e quindi sociale, tra vincitori e vinti. Pertanto, sulla base di questo ragionamento, è conseguentemente più che legittima la presenza dell'antico signum di cui parla Ovidio all'interno santuario ceriolense, ovvero di una statua dedicata alla dea in seguito alla distruzione di Falerii; ma l'iscrizione citata nei Fasti non può che documentarci semplicemente della provenienza della statua, e non del culto della Minerva del Celio.

Pertanto, se prendiamo in considerazione le caratteristiche proprie del rituale dell'evocatio, così come abbiamo evidenziato, ed inoltre se consideriamo la rigida concezione del rapporto uomo-divinità che regolava la vita religiosa dei Romani, una relazione consistente essenzialmente in un foedus, fondamentale per il benessere e la salvezza della comunità, ma rotto il quale si rischiava di provocare l'ira deum ${ }^{67}$, credo che si possa abbandonare senza più dubbio alcuno la teoria di una 'cattività' della dea. Ad ulteriore supporto della infondatezza di questa interpretazione, dobbiamo per di più aggiungere una seconda e fondamentale considerazione: in effetti, dalle fonti, conosciamo con certezza soltanto due evocationes, quella di Iuno Regina da Veio del 396 a.C. ${ }^{68}$ e di Iuno Caelestis da Cartagine nel 146 a.C. ${ }^{69}$; una terza evocatio, infine, potrebbe essere documentata epigraficamente anche per la città turca di Isaura Vetus, per 1'anno 75 a.C. circa $^{70}$. Quindi, in generale, siamo di fronte ad una inspiegabile scarsezza di eventi, se paragonati numericamente a quello delle città conquistate dai Romani; verosimilmente, però, l'unica giustificazione plausibile per un numero così ridotto di evocationes certe deve risiedere probabilmente solo nella mancanza di testimonianze documentarie in merito: invero, la letteratura moderna, sulla base sia di attendibili ipotesi supportate da comprovati dati storici, sia di supposizioni invece non del tutto fondate, ha in realtà ritenuto di poter individuare in numerose altre occasioni delle divinità evocate ${ }^{71}$. Ora, esaminando i casi di evocatio, in nessuno di essi, né in quelli concretamente documentati, né tanto meno nei molteplici casi più o meno correttamente ipotizzati, conosciamo una divinità definita 'prigioniera', una divinità 'catturata' in quanto evocata; ovvero, nessun altro dio, concretamente o solo verosimilmente portato a Roma in seguito a tale rituale, nel lungo corso della storia romana, ha 'meritato' l'epiteto di captus/a, la qualifica di divinità 'presa': questo perché, è evidente, non si trattava di divinità catturate, bensì di divinità che avevano manifestato il loro accordo ad integrarsi nel pantheon romano. Quindi, in definitiva, l'esempio della Minerva ceriolense costituirebbe un unicum, decisamente

67 È il rischio di alterazione della religio, del corretto modo di compiere il rituale e di venerare gli dei, alla base del sentimento religioso romano; ovvero, "the correct form of "social' relations with the gods, essentially by celebrating the rituals implied by the links that exist between the gods and men" (Scheid 2003, 23): celebri ed indicative a questo riguardo sono le numerose citazioni ciceroniane sulla religio, dapprima definita, in opposizione alla superstitio, come ciò quae deorum cultu pio continetur (Nat.D., I, 117); ma la religio è anche eam, quae in metu et caerimonia deorum sit, appellant (De Invent., II, 66), meglio delineata qualche paragrafo più avanti come quae superioris cuiusdam naturae, quam divinam vocant, curam caerimoniamque affert (De Invent., II, 161).

68 Liv., V, 21, 1-3; V, 22, 3-7; Val. Max., I, 8, 3; Plut., Cam., VI, 1-2.

69 Macr., Sat., III, 9, 1-9; Serv., in Aen., XII, 841.

70 Gustafsson 2000,60ss.; Ferri 2010b. Di questa città non conosciamo il nome della divinità, evocata con la generica ma rituale formula sive deus sive dea.

71 Almeno un paio di questi casi sono più che verosimili, in particolare quelli di Iuno Curitis da Falerii e di Voltumna da Volsinii. Per un elenco completo delle varie proposte di identificazione di ulteriori evocationes cfr. Ferri 2010a, 155 n. 1 . 
troppo insolito per essere credibile, ed oltretutto troppo 'sacrilego' per un popolo che del rispetto verso gli dei aveva fatto uno dei motivi principali del proprio successo.

Ma rifiutando la lettura di capta come 'prigioniera', a nostro avviso viene di conseguenza a mancare anche il nesso tra il santuario del Celio e la conquista di Falerii; ovvero, in altri termini, se Minerva non è più una divinità 'catturata', se l'attributo di capta non le viene assegnato in seguito all'evocatio, a questo punto non è più necessario neppure ipotizzare che il culto della dea provenisse dalla città falisca. Ovidio, infatti, dobbiamo tornare a ribadirlo, nel tentativo di giustificare un epiteto dal significato ormai oscuro, propone una provenienza falisca sulla base di un'iscrizione antica che poteva leggere all'interno del santuario, un'iscrizione apposta verosimilmente su di una statua che poteva essere stata benissimo presa da Falerii e portata a Roma ex manubiis ${ }^{72}$; ovvero, "afferma soltanto che l'epiclesi Capta potrebbe alludere alla provenienza forzata da Falerii" ${ }^{\prime 3}$, senza però dare per certa questa informazione. Pertanto, l'ambiguità dell'espressione ovidiana, forse peraltro voluta in questa circostanza, come ipotizza Torelli ${ }^{74}$, relativamente a questa iscrizione, non ci permette di accogliere il signum e la prisca littera come una testimonianza sicura dell'origine falisca del culto; conseguentemente, non possiamo considerare questa come prova sufficiente per credere ad una 'estraneità' del culto ceriolense rispetto al pantheon romano, ad una origine straniera della Minerva Capta ovidiana ${ }^{75}$.

\section{Una dea 'sposata'?}

Avendo dimostrato l'inesattezza della proposta esegetica tradizionale, ossia avendo, per così dire, 'emancipato' la Minerva Capta da una presunta quanto improbabile 'prigionia', quale dobbiamo ritenere, dunque, la corretta lettura dell'epiteto Capta? Come già ricordato, $i$ tentativi di interpretazione sono stati molteplici; ed il primo ad allontanarsi dal 'solco della tradizione' fu Radke, che giunse a supporre, in maniera

72 L'esistenza di un importante culto dedicato a Minerva nella città di Falerii è testimoniato, oltre che dalle fonti letterarie, probabilmente anche da moderni ritrovamenti archeologici: infatti, recentemente è stata proposta una convincente identificazione del luogo di culto della dea con i resti monumentali rinvenuti in località 'Lo Scasato' (Moretti Sgubini 1998, 56ss.); notizie sui ritrovamenti fittili dell'area, note da tempo, sono state solo negli anni '80 correttamente interpretate (Moscati 1990, 163s.) e successivamente confermate da ulteriori indagini, che hanno portato alla luce reperti databili ancora al V sec. a.C. (Moscati 1990, 162ss.; De Lucia Brolli 2003, 65 ss.). Quello che deve essere considerato il primo tra i templi urbani di Falerii, al momento è soltanto in forma ipotetica attribuibile a Minerva: infatti, nella quasi totale incertezza dei dati archeologici (unico dato a favore di questa interpretazione è il rinvenimento di alcuni altorilievi, di grande qualità tecnica ed artistica, degli inizi del IV sec. a.C., tra i quali si distinguono le figure di Giove, Giunone ed appunto Minerva - Colonna 1992, 108ss.), si deve considerare che dalle fonti antiche conosciamo per Falerii i culti di Minerva e di Giunone Curite; ora, dal momento che quest'ultimo è stato individuato con certezza nell'area extra-urbana di Celle, meglio indicata per un culto federale, è stato ipotizzato che il tempio dello Scasato fosse dedicato proprio alla nostra dea: invero, secondo la communis opinio, Minerva avrebbe avuto nella città falisca un culto poliadico, e pertanto un'area sacra a lei dedicata potrebbe ben essere individuata in un contesto urbano.

73 Pedroni 2011, 48

74 Torelli 1984, 53.

75 Però, come abbiamo visto, la tradizione annalistica in realtà conosceva, con indiscutibile certezza, una divinità evocata da Falerii; diversamente, dovremmo pensare ad una completa invenzione ovidiana della Minerva falisca portata a Roma. Ora, per risolvere questa impasse di una divinità evocata ma priva di culto a Roma, avendo escluso il tempio del Celio, credo che si debba seguire la proposta di Torelli, per il quale il tempio dedotto in seguito alla conquista del centro falisco deve riconoscersi nella Minerva in Aventino, "di cui non conosciamo l'anno di fondazione, ma che, come altri templi aventinesi ha larghe probabilità di essere stato costruito proprio nel III sec. a.C." (Torelli 1984, 53). Dello stesso avviso è anche Pedroni (Pedroni 2011, 41 e 48s.). 
decisamente originale ma priva di qualsiasi base documentaria, che Capta fosse il vero nome della divinità, e che invece Minerva fosse soltanto un suo attributo ${ }^{76}$; però, per quanto riguarda l'etimologia vera a propria del termine, le soluzioni esposte dal filologo tedesco non si discostano da quelle solite, che vi vedono o una dea 'prigioniera' (da capere), o una dea dell'ingenium (da caput).

La vera 'rivoluzione copernicana' nell'analisi della figura di Minerva Capta si deve però alla fondamentale opera monografica su matrimonio e riti iniziatici di Torelli ed all'articolo di Coarelli sul tempio del Celio ${ }^{77}$ : il primo, infatti, in base ad un minuzioso e dettagliato esame delle relazioni calendariali e cultuali tra la Minerva del 19 marzo $^{78}$ e le altre festività del primo mese dell'anno arcaico, in particolare con Anna Perenna e con le feste del ciclo dei Salii, giunge alla conclusione che Minerva fosse titolare dei rituali d'iniziazione femminile e di quelli connessi con le nuptiae ${ }^{79}$; però, seppur identificando e definendo le competenze di Minerva Capta, non ne chiarisce etimologicamente l'epiteto. A questo proposito si è invece accinto Coarelli, in un lavoro di poco posteriore, il quale, sulla scorta del lavoro di Torelli, è giunto ad interpretare l'aggettivo capta in maniera totalmente differente rispetto alla precedente tradizione: sempre con il significato di 'presa', ma in questo caso nell'accezione di 'sposata'80; 1'analisi di Coarelli, invero, si basa su di un accurato esame della mitografia relativa alla dea, considerata sinotticamente insieme alle connessioni calendariali e rituali evidenziate da Torelli per il mese di marzo. In effetti, l'unico mito prettamente romano in cui interviene Minerva è quello raccontatoci da Ovidio nei Fasti, a proposito delle idi di marzo e della festa di Anna Perenna ${ }^{81}$ : Marte, invaghitosi di Minerva, avrebbe interpellato Anna per chiederle aiuto nel tentativo di conquistare la sua amata; ma la vecchia Anna, invece di lasciarsi convincere dal dio, se ne prende apertamente gioco, sostituendosi a Minerva e presentandosi in vesti da sposa al momento delle nozze: si tratta del cosiddetto mito delle 'false nozze',

76 Radke 1965, 81: "da eigentlich Minerva der Beiname für Capta ist; der volle Name lautet Capta Minerva." Segue la sua proposta anche Canciani (Canciani 1984, 1075).

77 M. Torelli, Lavinio e Roma. Riti iniziatici e matrimonio tra archeologia e storia, Roma, 1984; F. Coarelli, "Il tempio di Minerva Capta sul Celio e la domus di Claudio”, RPAA 70 (1997-98) 209ss.

78 Il 19 marzo si celebrava la festa delle Quinquatrus, la festività principale di Minerva sin dall'epoca arcaica, come testimoniato dai Fasti Antiates Maiores (Degrassi, Inscr.It., XIII.2, pp. 6-7). In merito alla titolarità delle Quinquatrus a Minerva sin dalle origini di tale celebrazione, vd. T. Cinaglia, "Le Quinquatrus, una festa di Minerva", Gerión 34 (2016), c.d.s.

79 Torelli 1984, 50ss.; idem 1990. Teoria successivamente ribadita dalla Massa-Peirault (Massa-Peirault 1987, 206ss.) e da Coarelli (Coarelli 1998, 214); cfr. in merito Enea nel Lazio, il catalogo della mostra sui rinvenimenti effettuati nel santuario di Minerva a Lavinio.

80 Coarelli 1998, 214. Successivamente di questo parere anche la Mezzetti (Mezzetti 1997-2000, 185s.).

81 Ov., Fast., III, 675-696. Già a cavallo tra Ottocento e Novecento, però, alcuni studiosi arrivarono a sostenere che il matrimonio tra le due divinità, e più in generale tra gli dei romani, non fosse una caratteristica genuina, ma che si dovesse attribuire ad una tarda influenza dovuta alla interpretatio greca (Aust 1889, 19ss.; Warde Fowler 1899 , 59ss.; Wissowa 1902, 26ss.); e sono tuttora numerosi gli assertori di una origine greca del mito, con protagonisti Ares ed Atena, che sarebbe stato successivamente rivisitato in chiave romana da poeti ed eruditi in epoca imperiale (Evans 1939, 159ss.; Scullard 1981, 248 n. 114; Hinds 1992, 100s.: sostiene che si tratti di uno 'script', di un copione drammatico; Schilling 2003, 161 n. 232: afferma che sia una "invention fantaisiste d'Ovide"; Phillips 2006, 942). È stato anche sostenuto, inoltre, che la partecipazione di Minerva a questo mito sia da considerarsi 'tarda': in effetti, alcuni studiosi considerano la presenza della dea come il frutto di una sostituzione, in epoca tardo-repubblicana, a discapito di Nerio, che sarebbe stata invece la originaria protagonista delle nozze con Marte (Warde Fowler 1899, 60; Hild 1969c, 802 n. 8; Frazer 1973, 121ss.). Particolare, infine, è l'interpretazione del rapporto tra Marte e Nerio teorizzata da Usener e Aust, secondo i quali il matrimonio di Marte nel suo mese rappresentava, nelle nozze con la giovane Nerio, la celebrazione del nuovo anno, mentre la coppia Anna Perenna - Mamurius Veturius, al contrario, avrebbe indicato la certificazione della fine del vecchio anno (Usener 1875 , 221ss.; Aust 1899, 132s.). 
dal chiaro valore apotropaico, finalizzato ad allontanare il male dalla $n u b e n d a^{82}$. Le notizie circa l'intricato matrimonio di Marte sono completate da Aulo Gellio ${ }^{83}$, il quale, riportando alcuni versi del Truculentus di Plauto, ci informa di una seconda tradizione mitografica, per la quale il dio sarebbe convolato a giuste nozze con Nerio, divinità sabina, nella quale la vulgata moderna è concorde nel riconoscere la fortis dea cui si deve sacrificare il giorno del Tubilustrium, il 23 marzo ${ }^{84}$; Marte, dunque, terminato il periodo della lustratio del suo mese, può finalmente sposare Minerva-Nerio ${ }^{85}$. Secondo Coarelli, inoltre, la connessione tra Minerva Capta ed il rituale matrimoniale è per di più ribadito dalla presenza, all'interno del succitato testo di Gellio, della preghiera di Ersilia, la sposa sabina di Romolo, che invoca Nerio affinché questa le conceda nuptiis propriis et prosperis uti, quod de tui coniugis consilio contigit uti nos ibidem integras raperent, unde liberos sibi et suis, posteros patriae pararent; pertanto, lo studioso conclude che "siamo in presenza di un aition delle più antiche nozze romane, quelle per raptus, che nel mito delle Sabine trovavano l'altro modello ancestrale" $" 86$ e che questo santuario, pertanto, doveva essere consacrato ad una 'Minerva sposata', una Minerva che diventa la coniuge di Marte sotto le spoglie di Nerio. Secondo questa esegesi, dunque, l'aggettivo capta è da intendersi col significato di 'presa' e quindi, per traslato, di 'sposata'; una accezione che, secondo Coarelli, doveva avere per di più valore formulare, facendo così del verbo capio il termine tecnico del più antico rito delle nozze, quello per raptus, per l'appunto ${ }^{87}$.

Ma se ormai costituiscono un punto fermo, per l'indagine del culto di Minerva, le conclusioni raggiunte da Torelli e Coarelli sulle connessioni religiose e cultuali del più antico feriale romano tra la nostra dea e le altre divinità del mese di marzo,

82 È una prassi presente in numerose culture primitive, documentata inoltre da studi antropologici anche per l'epoca moderna (Frazer 1973, 125ss.; Boels-Janssen 1993, 35).

83 Gell., XIII, 23, 10-14.

84 Ov., Fast., III, 849-850: Summa dies e quinque tubas lustrare canoras / Admonet et forti sacrificare deae; Lyd., de Mens., IV, 60: Altheim, Bömer e Scullard attribuiscono in realtà il sacrificio del Tubilustrium a Minerva, e non a Nerio Martis, ipotizzando che la prima sia stata sostituita alla seconda in età tardo-repubblicana (Altheim 1932, 1779; Bömer 1957-58, II, 202; Scullard 1981, 94).

85 Gell., XIII, 23, 4: Te Anna ac Peranna, Panda Cela, te Pales, / Nerienes <et> Minerva, Fortuna ac Ceres.

86 Coarelli 1998, 213. Lo stesso Torelli arriva alla conclusione che il ratto delle Sabine indicasse simbolicamente l'istituzionalizzazione del più antico matrimonio, che diventa matrimonium iustum proprio a marzo, tra Quinquatrus e Tubilustrium, quando i Salii sono in azione, e la nubenda, la fanciulla 'rapita', poteva forse rivivere il proprio archetipo di 'Sabina' (Torelli 1984, 112s.).

87 Coarelli (Coarelli 1998, 213) ritiene di poter individuare un parallelo, a conferma della sua ipotesi, nella formula cerimoniale con cui il pontefice accoglieva la nuova vestale: infatti, secondo Gellio (I, 12, 14), il sacerdote riceveva quelle che erano poco più che bambine con le parole 'ita te, Amata, capio' (cfr. anche Gaius, Inst., I, 130). Ma la traduzione del verbo capio con il verbo 'sposare' risulta, a mio avviso, una forzatura, non esistendo documentazione ulteriore a garanzia di questa particolare accezione nell'uso del verbo e non essendoci i presupposti per individuare nel rapporto pontifex-vestalis una relazione 'coniugale', quantunque sacra (la Hersch ha correttamente evidenziato le varie differenze rituali e simboliche tra la captio della sposa e quella della vestale - Hersch 2101, 146ss.); meglio, in questo caso, seguire una interpretazione 'classica', traducendo più comunemente il verbo capio con il significato di 'prendere' (cfr. TLL, III, s. v. capio, 335, con 1'accezione di eligere, scegliere): al verbo capio, in effetti, era indubbiamente attribuito un valore formulare, in quanto impiegato ad indicare l'azione della scelta di un nuovo componente all'interno di un gruppo sacerdotale (e.g.: Serv., in Aen, VII, 303: sacerdotes creari, virgines capi dicimus). D'altronde, come ha ben spiegato Dumézil, per quanto concerne il termine amata, “il n'y a lieu de torturer l'étymologie, ni d'écrire le mot avec une majuscule (n.d.a.: per coloro che lo interpretano come un nome proprio), ni d'en déduire aucune forme de tendresse, même stylisée, conjugale ou paternelle, entre la Vestale et le pontife" (Dumézil 1979, 242), perché questo aggettivo è da intendersi come una qualità della dea stessa, ovvero di Vesta, di cui la sacerdotessa è l'incarnazione: pertanto, è la dea stessa "qui définissaient à la fois la condition d'âge de la nouvelle prêtresse et la qualification qui lui était donnée lors de sa captio: toute jeune, et amata." (Dumézil 1979, 241ss.). Sul rituale delle nozze per raptus cfr. Dumézil 1979, 73ss.; Torelli 1984, 75ss., 117 ss. (sempre Dumézil individua un parallelo delle nozze per raptus nei rituali indiani - ibidem, $67 \mathrm{ss}$.). 
relazioni oltretutto ribadite anche dalla mitografia di stampo non-ellenizzante in cui essa risulta coinvolta, non reputo d'altro canto accettabile l'ipotesi che fa della Minerva Capta una divinità 'sposata': infatti, per quanto banale possa risultare ad un primo approccio tale osservazione, non è Minerva che sposa Marte, bensì la sabina Nerio. Ma cercherò di spiegarmi meglio e di motivare questa affermazione. Il mese di marzo, è noto, rappresenta un generale periodo di purificazioni, di lustrationes, nell'ottica di una preparazione del mondo naturale e umano alla riproduzione ed alla partenza per la guerra; ma costituisce anche un periodo di sconvolgimento, di ribaltamento dell'ordine sociale, all'inizio del nuovo anno, vòlto al rinnovamento ed alla ricostituzione dello stesso ${ }^{88}$. Ora, questa fase di 'sospensione' temporanea dell'ordine precostituito e di purificazioni termina il 23 marzo, giorno del Tubilustrium, quando, dopo l'ultimo intervento dei Salii, in seguito alla purificazione dei tubi quibus in sacris utuntur ${ }^{89}$, finalmente possono avere inizio le campagne militari; ed a partire dal 24, infine, è altresì possibile celebrare le nozze: invero, sino a questa data era fatto espresso divieto di contrarre matrimonio, essendo evidentemente proibito durante il lungo periodo lustrale ${ }^{90}$. Non credo, pertanto, sulla base di questi presupposti, che il matrimonio tra Marte e Nerio sia solo una mera trasposizione sotto altre vesti della mitografia relativa a Minerva; ritengo, piuttosto, che se il dio può convolare a giuste nozze solo il 23 del mese, è chiaramente perché questo è l'ultimo giorno del periodo di purificazioni, momento in cui finalmente cade il divieto di sposarsi; e, allo stesso modo, ritengo che le mancate nozze tra Marte e Minerva non siano solo un topos letterario e che quindi non abbiano soltanto un valore apotropaico, come abbiamo precedentemente sottolineato, ma che al contrario indichino anche e soprattutto l'impossibilità, ancora alla metà del mese, di poter contrarre matrimonio: d'altronde, come messo in evidenza da Moret, le nozze legittime (nuptiis propriis) richieste da Ersilia alla dea Nerio lasciano supporre che questo divieto debba ancora cadere nel giorno del Tubilustrium ${ }^{91}$. Tra il 15 ed il 19 di marzo, pertanto, Marte non può sposare Minerva, ma sarà la sua alter ego sabina a sposare il dio, quasi alla fine del mese, il giorno 2392; il rifiuto di celebrare le nozze, trasposto sul piano comico

88 Tutto il periodo in cui intervengono i Salii è un momento di purificazione, all'inizio del nuovo anno, per la comunità romana ( $c f r$. Torelli 1984, 50ss.; idem 1990, 99ss.).

89 Degrassi, Inscr.It., XIII.2, Fasti Praenestini, tav. XXXVIII. Le altre fonti sul Tubilustrium sono quelle di Varrone (Ling.Lat., VI, 14): Dies Tubilustrium appellatur, quod eo die in Atrio Sutorio sacrorum tubae lustrantur; e di Festo (480L): Tubilustria, quibus diebus adscribtum in $<$ Fastis est, in Atr $>$ io Sutorio agna tubae $<$ lustrantur, quos $>$ tubos appellant; quod genus <lustrationis ex Ar $>$ cadia Pallanteo trans $<$ latum esse dicunt. $>$

90 Torelli 1984, 112s.; Hersch 2010, 47. Ov., Fast., III, 393-394: Nubere siqua volens, quamvis properabitis ambo, / differ; habent parvae commoda magna morae.; Porph., Epist., II, 2, 209: ob quam rem Maio mense religio est nubere, et item Martio, in quo de nuptiis habito certamine a Minerva Mars victus est, et obtenta virginitate Minerva Neriene est appellata. Più in generale, sui giorni non propizi o in cui era espressamente vietato contrarre matrimonio, $c f r$. Hersch 2010, 44ss.

91 Moret 2012, 93.

92 Che sia Nerio la paredros di Marte, e non Minerva, è inoltre comprensibile dalle caratteristiche della divinità stessa: 1'epiclesi della dea, Nerio Martis o Neriene (Enn., Ann., fr. 99 Skutsch; Gell., XIII, 23; Lyd., de Mens. IV, 60; Porph., in Hor. epist., II, 2, 209; Mart. Cap., I, 4), la qualifica come personificazione della forza e del valore guerriero del dio (Dumézil 1953, 182s.); ma ancor più importante è l'esame dell'aspetto linguistico, per questa divinità di origine sabina (Wissowa, s.v. Nerio, in Roscher, III.1, 271; Dumézil 1953; Pighi 1958; Sabbatucci 1988, 139ss.): infatti, il nome stesso della dea, la cui radice indo-europea è *ner-, ci conduce al dualismo *ner- / *viro-, ossia all'opposizione 'uomo con funzione guerriera' / 'uomo con funzione civile'; e pertanto, il termine Nerio ci indirizza verso una realtà fortemente connotata da caratteristiche militari, che ben si addice alla compagna di Marte. 
dalla mitografia nella vicenda delle 'false nozze' con Anna Perenna ${ }^{93}$, deve pertanto necessariamente corrispondere anche ad uno stringente divieto sul piano cultuale, per quanto riguardava la arcaica comunità romana ${ }^{94}$. Ed è a motivo di ciò, in conclusione, che reputo inverosimile pensare al culto di Minerva Capta come a quello di una dea 'sposata': in quanto divinità la cui festa ricorre ancora nel periodo di interdizione delle nozze, in quanto dea che in realtà sfugge allo sposo Marte, mandando al suo posto la vecchia ed irridente Anna Perenna, Minerva e le celebrazioni del 19 marzo a lei connesse non potevano in alcun modo rappresentare la ritualizzazione del matrimonio ${ }^{95}$.

\section{Una dea 'accolta'; o piuttosto, una dea 'che prende'}

Sono state pertanto confutate le ipotesi di una Minerva 'prigioniera' e di una Minerva 'sposata'. Vi è anche un'ulteriore esegesi dell'epiteto Capta, recentemente esposta dalla Gustafsson, per la quale il verbo capio sarebbe da interpretare con il valore di 'accettare', teorizzando, quindi, una 'Minerva accettata' ${ }^{96}$; questa proposta, però, non viene 'teologicamente' giustificata dalla storica svedese, che lascia così in sospeso il tema centrale e scopo ultimo dell'analisi, ovvero risalire all'origine dell'appellativo per comprendere le forme del culto tributato alla dea. In conclusione, dunque, quale valore si deve attribuire all'epiteto Capta? Credo che la risposta sia stata già individuata, anche se sulla base di presupposti errati, in un lavoro relativamente recente del filologo tedesco Köves-Zulauf ${ }^{97}$; in effetti, pur tornando a riproporre la tradizionale origine falisca per il culto del Celio, inaccettabile se non si fa più di Minerva una 'dea prigioniera', lo studioso elabora una interpre-

93 Va sottolineato che la festa di Anna Perenna cadeva il 15 marzo, e quindi in stretta connessione calendariale, oltre che mitografica, sia con Minerva che con Marte.

94 Come d'altronde evidenziato, quasi un secolo e mezzo fa, già da Usener (Usener 1875, 221ss.). Da questo punto di vista, non è pienamente condivisibile l'affermazione di Carandini, per il quale "il 19 marzo, alle Quinquatrus, Marte sposa Neriene e dà vita all'istituzione matrimoniale" (Carandini 2006, 344): se infatti è vero che le nozze tra Marte e Nerio certificano ormai la possibilità di contrarre matrimonio, questo però non è ancora possibile il 19 di marzo, ma soltanto 4 giorni più tardi, al termine del periodo lustrale, dopo il Tubilustrium, giorno in cui si sacrificava alla fortis dea Nerio.

95 Una ulteriore prova a supporto di questa interpretazione credo sia rintracciabile in un passo di Servio, sempre trascurato dalla letteratura moderna (l'unico autore che lo menziona, senza tentarne però una spiegazione, è Hild - Hild 1969c, 802 n. 3): si tratta di un brano del Commentarius in Georgicon Librum (I, 277) che così recita: Quintam fuge: dicitur enim hic numerus Minervae esse consecratus, quam sterilem esse constat. Le implicazioni di questo testo sono decisamente molteplici, ma in questa sede dobbiamo limitarci ad una sola osservazione puntuale, pertinente al nostro discorso: infatti Minerva viene detta 'sterile', caratteristica che evidentemente nulla ha a che vedere con l'attributo di 'vergine' (virgo e virginitas occorrono nei seguenti autori in relazione alla dea: Amp., IX, 10; Cic., Nat.D., III, 59; Ov., Met., IV, 754; Porph., in Hor. Epist., II, 2, 209; Verg., En., XI, 483) con cui spesso è associata la nostra dea; non è possibile entrare nel dettaglio della spiegazione, ma ritengo comunque che sia possibile ricondurre la sterilità di Minerva proprio alla collocazione calendariale delle sue Quinquatrus, all'interno del periodo lustrale, connessa con la prima mestruazione femminile ( $c f r$. Torelli 1984, 73s.). In questa sede, però, ritengo basti osservare che mal si addice ad una dea 'sposata' una condizione di sterilità, essendo la fertilità il presupposto necessario del matrimonio, il cui unico scopo era quello di istituzionalizzare un rapporto vòlto alla procreazione, finalizzato unicamente alla continuità della comunità. Sul passo di Servio e sul tema della sterilità di Minerva vd. più in dettaglio T. Cinaglia, "Le Quinquatrus ed il numero 5, ovvero la correlazione tra Anna Perenna e Minerva nei rituali d'iniziazione femminile”, Museum Helveticum (2018/1), c.d.s.

96 Gustafsson 2000, 58.

97 Successivamente seguito nella sua interpretazione da Ferri: entrambi gli studiosi, pur negando il tradizionale significato di 'prigioniera' all'aggettivo capta, continuano ad associare Minerva Capta alla conquista di Falerii del 241 a.C. (Köves-Zulauf 1993, 159; Ferri 2006, 231). 
tazione totalmente differente rispetto alle precedenti teorie sull'epiclesi della dea: la fondamentale novità esegetica risiede nel 'rovesciare' il valore del verbo, nel renderlo da passivo ad attivo ${ }^{98}$; successivamente Ferri, accogliendo questa lettura, giunge a sostenere che "Capta starebbe di conseguenza per: 'l'accogliente" Ovvero, non avremmo più una divinità che 'subisce' l'azione espressa dal verbo, non più quindi una dea 'catturata' o 'sposata', bensì saremmo di fronte ad una divinità che è essa stessa la causa incipiente dell'azione; e l'analisi, metodologicamente e filologicamente corretta, si basa su varie altre occorrenze di casi simili all'interno del pantheon romano arcaico, assolutamente noti ed incontrovertibili: lo studioso tedesco, in effetti, riporta tre esempi di desinenza passiva avente però valore attivo, sempre per epiteti pertinenti a divinità femminili, ossia quelli di Iuno Februata, Mater Matuta e Fortuna Viscata ${ }^{100}$, tutte divinità di notevole arcaicità, documentate per lo meno a partire dal VI sec. a.C. ${ }^{101}$ Come afferma Ferri, "queste divinità hanno compiuto le azioni inerenti al loro epiteto, le possono sempre compiere e le compiranno"102; nello specifico, februata è 'colei che purifica', matuta è invece 'colei che fa maturare' (o 'colei che fa del bene'), mentre viscata è infine 'colei che invischia' ${ }^{\prime 03}$. Ed a questo elenco dobbiamo inoltre aggiungere, tralasciati dai due autori, altri due casi analoghi, ovvero quello della celeberrima Iuno Moneta e quello di Iuno Sospita ${ }^{104}$, la prima una dea 'che ammonisce' e la seconda una dea 'che salva'105; divinità anch'esse molto risalenti, pertinenti ancora ad una fase arcaica della

98 "Es bietet sich jedoch ein naheliegender Ausweg, wenn man das Wort nicht als ein Gebilde gewöhnlicher klassischer Latinität versteht, sondern als archaische Bildung; wenn man der Endung -ta statt der klassischen passiven aktive Bedeutung zuschreibt" (Köves-Zulauf 1993, 164).

99 Ferri 2006, 233s. A questo riguardo $c f r$. anche TLL, III, s. v. capio, 335.

100 Köves-Zulauf 1993, 164. Le fonti su Mater Matuta sono innumerevoli (cfr. elenco in Hild 1969b, 1625s.; Pisani Sartorio 1995, 281ss.), ma particolarmente importante è il testo di Festo (159L): Mater Matuta: manis, mane, matrimonium, mater familiae, matertera, matrices, materiae dietae videntur, ut ait Verrius, quia sint bona, qualia scilicet, quae sunt matura, vel potius a matre, quae est originis Graecae. Mentre per le altre due epiclesi le fonti note sono rispettivamente, di nuovo, Festo nell'epitome paolina (60L): Februarius mensis dictus, quod tum, id est extremo mense anni, populus februaretur, id est lustraretur ac purgaretur, vel a Iunone Februata, quam alii Februalem, Romani Februlim vocant; e Marziano Capella (II, 149): nam Fluoniam, Februalemque ac Februam mihi poscere non necesse est, quum nihil contagionis corporeae sexu intemerata pertulerim. Infine, per quanto concerne Viscata, Plutarco è l'unico che ci tramanda suddetto epiteto (Quest.Rom., 74, 281e):

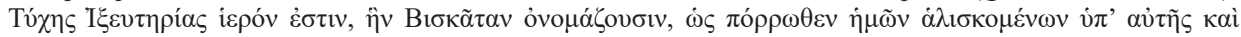

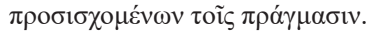

101 Iuno Februata (o Februalis) è connessa con la celebrazione della festa dei Lupercalia il 15 febbraio, celebrazione arcaica in cui Giunone sovrintende ai rituali di purificazione (da februa) ed al parto (Hild 1969a, 686; Dury-Moyaers - Renard 1981, 154s.; Boels-Jannsen 1993, 303s. e 423). Mater Matuta è invece venerata durante la festa dei Matralia dell'11 Giugno, anch'essa antichissima, ed il suo epiteto è messo in rapporto con l'Aurora e con matutinus, maturus ('del mattino', 'maturo',) o con manus, mane ('buono'), in qualità di dea del 'Giusto Tempo' o di 'Buona Madre'; il suo tempio sarebbe stato dedicato, secondo la tradizione, da Servio Tullio, ma in realtà il culto della dea deve rimontare ad un'epoca ancora più arcaica, poiché la leggenda attribuisce la sua introduzione a Roma a Numa (Dumézil 1966, 66ss.; Hild 1969b, 1625; Champeaux 1982, 307ss.; Bouma 1992; Pisani Sartorio 1995, 285 con bibliografia aggiornata). Per quanto riguarda Fortuna Viscata, infine, si tratterebbe di una dea 'che invischia', 'che irretisce' gli uomini; il suo culto sul Palatino sarebbe stato fondato, secondo la tradizione, da Servio Tullio (per una amplia bibliografia $c f r$. Champeaux 1982, 411ss.; Aronen 1995, 280s.).

102 Ferri 2006, 233 n. 197.

103 Ernout - Meillet 1932, 222, 412 e 741; Walde-Hofmann 1938, I, 472; II, 53s. e 801; de Vaan 2008, 208 , 367 e 683.

104 Le fonti su Iuno Moneta sono varie (per un elenco esaustivo cfr. Giannelli 1996b, 123ss.); lo stesso dicasi di Iuno Sospita (per un elenco completo $c f r$. Hild 1969a, 686s.), però è particolarmente rilevante la testimonianza di Festo (510L), che ci tramanda il valore dell'epiclesi: Sispitem Iunonem, quam vulgo sospitem appellant, antiqui usurpabant, cum ea vox ex Graeco videatur sumpta, quod est $\sigma \omega ́ \zeta \varepsilon 1 v$.

105 Ernout - Meillet 1932, 391 e 638; Walde - Hofmann 1938, II, 108 e 564s.; de Vaan 2008, 387 e 577. 
religione romana ${ }^{106}$. Siamo, orbene, di fronte a cinque epiteti formati da participi con diatesi passiva ma con documentato valore attivo; cinque divinità femminili di certificata arcaicità nella comunità romana: ritengo, di conseguenza, che non sia azzardato supporre che anche le origini della nostra Minerva Capta, sia in riferimento all'epiclesi, sia per quanto concerne evidentemente il culto stesso, debbano rimontare notevolmente lungo il corso della storia romana; con tutta probabilità direttamente all'epoca arcaica, divinità coeva alle altre sopra menzionate ${ }^{107}$.

Però, come precedentemente accennato, il presupposto che Minerva fosse comunque una divinità 'straniera' conduce i due studiosi a delle conclusioni, a mio avviso, discutibili e solo parzialmente accettabili: infatti Köves-Zulauf, nuovamente seguito da Ferri, sostiene che l'epiteto Capta fosse proprio di Minerva già a Falerii, quindi ben prima del supposto trasferimento della dea nel santuario ceriolense; qui, nel centro falisco, Minerva sarebbe stata una generica 'dea dell'accoglienza', la cui tutela si sarebbe rivolta alle "bande che, muovendosi di città in città, ne ottenevano anche con forza l'ammissione"108. Ma, accettando l'interpretazione classica di un culto poliadico della dea nella città falisca, il filologo tedesco va incontro, nella sua esposizione, ad una evidente incongruenza: non si comprende, infatti, come la Minerva di Falerii, con un ruolo di 'accoglienza' verso gli stranieri, ovvero con un culto che verosimilmente si doveva caratterizzare come liminare, marginale, potesse al contempo essere anche una divinità poliadica, dalle forme di culto palesemente in antitesi con il precedente. Però, come è stato già dimostrato, non è in alcun modo necessario pensare ad una Minerva falisca se si elimina il nesso capta-prigioniera sul quale Ovidio costruiva la sua paretimologia; conseguentemente, non è tanto meno necessario ipotizzare una divinità che accolga gli stranieri pur essendo poliadica. La funzione di 'accoglienza' della dea, la sua epiclesi di Capta, dunque, doveva estrinsecarsi 'semplicemente' nel ricevere, come affermano correttamente entrambi gli studiosi ${ }^{109}$, i giovani nel passaggio all'età adulta, al momento "der zweiten, der sozialen Geburt" ${ }^{110}$, ovvero al momento del passaggio di status

106 Di Iuno Moneta conosciamo il celebre tempio sull'Arx, dedicato nel 344 a.C., ma il suo culto deve essere sicuramente più risalente, dal momento che esisteva sull'Arce un naos dedicato alla dea già al tempo dell'assedio gallico; il culto è connesso con l'istituzione dell'officina monetale, ma l'epiteto, nello specifico, era evidentemente legato alle capacità oracolari della dea (Hild 1969a, 686s.; Dury-Moyaers - Renard 1981, 165ss.; Giannelli 1996b, 124). Di Iuno Sospita sappiamo invece di un tempio a Lanuvio, dedicato nel 338 a.C., e di due santuari cittadini, il primo del Foro Olitorio, dedicato nel 197 a.C., ed il secondo sul Palatino; il culto era celebrato alle calende di febbraio (Hild 1969a, 687s.; Boels-Jannsen 1993, 413ss. e 472s.).

107 Tralasciando per il momento la nostra interpretazione di Capta come 'colei che prende', con un valore attivo del verbo, le altre cinque attestazioni restano però certe ed indubitabili e pertanto documentano, per l'epoca arcaica, di un particolare utilizzo della forma passiva del participio passato; ma in realtà, questo non deve meravigliare particolarmente, perché ancora in epoca storica "certains participes passés s'emploient avec un sens actif, par ex. cautus, 'qui est sur ses gardes' de caveo; scitus 'qui sait' de scio" (Ernout 1945, 345): infatti, il participio passato latino si forma direttamente dall'unione della radice verbale ad un morfema *-to-, creando un aggettivo verbale con valore di compimento dell'azione; originariamente, infatti, "l'adjectif en *-to- qualifie un individu comme participant d'un procès" (Monteil 1970, 347), ovvero "indiquait que le sujet avait la qualité exprimée par le verbe; il pouvait avoir le sens actif aussi bien que le sens passif” (Ernout 1945, 344). Il latino poi, più di altre lingue in cui ugualmente fu produttivo questo suffisso, legò strettamente l'aggettivo in *-to- al verbo, da un lato aggiungendo spesso questo morfema al tema del perfectum, dall'altro associando al verbo l'aggettivo in *-to- per costituire la flessione perifrastica del medio-passivo nei tempi del perfectum: quindi, da una primitiva valenza di 'compimento totale dell'azione', si passò ad un valore perfettivo dell'aggettivo verbale; mentre il senso di compimento dell'azione nell'oggetto, presupponendo la passività dello stesso, ne determinò sempre più il valore passivo.

108 Ferri 2006, 234. Cfr. anche Köves-Zulauf 1993, 164ss.

109 Köves-Zulauf 1993, 168s.; Ferri 2006, 234.

110 Köves-Zulauf 1993, 168. 
dall'infanzia alla maturità. Ma in realtà, la proposta dei due autori deve essere parzialmente riconsiderata, dal momento che il verbo capio non ha mai il significato di 'accogliere', 'ricevere', bensì sempre una connotazione fisica e concreta dell'atto del 'prendere': in conclusione, senza però stravolgere questa interpretazione, dobbiamo forse meglio ritenere la Minerva Capta una dea 'che prende', concretamente, ossia una divinità direttamente coinvolta nel processo del passaggio di status; una dea il cui compito è quello di 'scegliere' da un gruppo di persone - pueri - alcuni elementi, per inserirli in un nuovo gruppo - iuvenes -, ovvero quello di inserire all'interno della comunità i nuovi pubes e le nuove viri potentes 'prendendoli' quali nuovi membri tra i componenti della classe di età inferiore ${ }^{111}$. Possiamo quindi affermare che la Minerva Capta del Celio fosse una divinità la cui azione del capěre si estrinsecasse non in una ipotetica connessione con gli stranieri, bensì in relazione alla iuventas, prendendo le nuove donne ed i nuovi uomini per renderli parte integrante del corpo civico romano; ed il cerchio si chiude se torniamo ad esaminare i risultati raggiunti da Torelli nell'analisi delle relazioni cultuali del più antico feriale romano in cui è coinvolta Minerva: di fronte ad una dea la cui sfera di competenza era rivolta in primis all'iniziazione giovanile, non vi è certamente miglior appellativo, miglior definizione di quella che ne fa una 'dea che prende', una 'dea che sceglie', alla stregua del pontifex quando ritualmente 'prendeva' in seno al gruppo delle altre sacerdotesse la nuova Vestale ${ }^{112}$. Pertanto possiamo concludere che l'azione di Minerva Capta si sviluppasse limitatamente ai riti di passaggio, alle iniziazioni giovanili, rituali concentrati nel periodo dal 15 al 19 marzo con le feste di Anna Perenna, dei Liberalia e delle Quinquatrus ${ }^{113}$, momento durante il quale "spetta alla dea Minerva vegliare sui giovani in qualità di kourotrophos" $" 14$.

\section{Conclusioni}

Al termine di questa lunga disamina in merito ai due nomi che la tradizione ci ha conservato per il santuario del Celio, ritengo si possa ormai affermare con certezza che il culto ceriolense mostri tutti i connotati dell'arcaicità; entrambe le denomi-

111 Fest., 296L: Pubes et qui pubem generare potest. Is incipit esse a quattuordecim annis: femina a duodecim viri potens, sive patiens, ut quidam putant. Con queste due espressioni di carattere tecnico-giuridico, ovvero pubes e viri potens, la giurisprudenza romana indicava l'inizio dell'età adulta per i giovani romani; l'età indicata da Festo, in realtà, pare non abbia avuto una concreta corrispondenza con la prassi quotidiana, per lo meno per l'epoca repubblicana, quando sembra fosse consuetudine per i pueri accedere alla successiva classe di età a 17 anni; lo stesso dicasi per le puellae, la certificazione della cui maturità sessuale era evidentemente finalizzata alla capacità procreativa, ma che molto frequentemente giungevano al matrimonio ben prima dei 12 anni e della comparsa del menarca (sul tema cfr. Tafaro 1988).

112 Cfr. n. 87.

113 I giovani Romani, infatti, celebravano l'uscita dall'infanzia e l'ingresso nell'età adulta, e quindi nella vita pubblica, il 17 marzo, nel giorno dei Liberalia: era in questa occasione che il giovane uomo deponeva la toga praetexta e vestiva la toga virilis; la cerimonia si svolgeva nel Foro, dove i giovani si recavano insieme alle proprie famiglie: al mattino si iniziava con un sacrificio ai Lares e successivamente il giovane assumeva la toga virile e deponeva le insignia pueritiae (Paul. Fest., 32L; Ov., Fast., III, 775-790; Ps. Acr. Hor., Sat. I, 5, 65; Pers., II, 70 e V, 31; Plut., Quaest. Rom., 101); quindi, dopo essere stato presentato nel Foro (ductio in forum), il giovane terminava il suo percorso rituale salendo al Capitolium, sacrificando agli dei dello stato e pagando un tributo alla dea Iuventas, una prassi che secondo la tradizione sarebbe stata istituita da Servio Tullio (Dion. Hal., Ant. Rom., 4.15.5). Sul tema dei Liberalia: Hunziker 1969, 353; Torelli 1984, 21; idem 1990; Massa-Pairault 1987, 218ss.; Sabbatucci 1988, 103ss.; Miller 2002, 199ss.; Moret 2012, 92s.

114 Menichetti 1995, 91s. 
nazioni, una volta esaminate in dettaglio, non possono che ricondurci ad una età risalente della storia romana: il nome Minervium, ad un epoca in cui il santuario doveva essere probabilmente ancora l'unico culto dedicato a Minerva in ambito urbano; quello di Minerva Capta, ad una fase arcaica della lingua latina, quando la diatesi passiva aveva anche un valore attivo. L'inserimento di questo santuario nella lista degli Argei, l'ignoranza dell'antiquaria romana sulle origini dell'epiteto Capta, la presenza di Minerva nel mito e nel ciclo festivo-rituale di marzo insieme a Marte ed Anna Perenna, sono tutti ulteriori indizi dell'arcaicità del culto ceriolense; un culto che verosimilmente deve essere considerato come il primo, e per lungo tempo il più importante, della dea a Roma; un culto già presente nel VI sec. a.C., ma che può forse risalire ad un'epoca ancora precedente, verosimilmente a partire dal VII, in stretta relazione con la fondazione delle Curiae Novae e con l'ampliamento del corpo cittadino con i Latini dopo la conquista di Alba Longa ${ }^{115}$.

Credo, a questo punto, che sia stata definitivamente risolta l'aporia evidenziata inizialmente, dal momento che non si ha più una situazione ossimorica con due diversi nomi indicanti, per il medesimo santuario, due realtà indubbiamente distanti e confliggenti tra loro, la prima arcaica e la seconda medio-repubblicana; il primo nome svelandoci un background prettamente romano, con gli Argei, ed il secondo invece prefigurando una completa estraneità al pantheon romano. Perché siamo di fronte, per l'appunto, a due facce della stessa medaglia; a due lati, a due aspetti, molto diversi tra loro, senza dubbio, ma pertinenti ad una stessa realtà, ad una 'medaglia' certamente dagli indubitabili connotati arcaici ${ }^{116}$.

115 Torelli 1984, 55; Carandini 1997-2003, 575.

116 Espongo soltanto in nota una recentissima teoria sull'epiteto di Capta proposta da Ferri (Ferri 2010a, 125ss.; idem 2011), in quanto a mio parere del tutto priva di qualsiasi fondamento scientifico: l'autore, invero, dopo aver inizialmente appoggiato la teoria di Köves-Zulauf ( $c f r$. n. 97 e successive), rinnega successivamente questa sua iniziale interpretazione giungendo quindi a conclusioni di tutt'altro stampo: infatti, sempre partendo dall'assunto che la dea fosse originaria di Falerii, arriva a sostenere che l'aggettivo captus/-a debba far riferimento non più ad una dea 'che accoglie', bensì ad una dea 'incatenata', 'avvinta in catene', "secondo un uso ben attestato nel mondo greco e nel Vicino Oriente” (Ferri 2010a, 125). L'unica divinità romana citata come esempio in questa esposizione, in quanto l'unica effettivamente testimoniataci dalle fonti letterarie per il mondo romano, è Saturno, i piedi della cui statua nel tempio del Foro erano avvinti da corde di lana, che venivano annualmente sciolte durante i Saturnalia (Macr., Sat., I, 8, 5: Cur autem Saturnus ipse in compedibus visatur, Verrius Flaccus causam se ignorare dicit: verum mihi Apollodori lectio sic suggerit. Saturnum Apollodorus alligari ait per annum laneo vinculo, et solvi ad diem sibi festum, id est mense hoc Decembri: atque inde proverbium ductum, deos laneos pedes habere: significari vero decimo mense semen in utero animatum in vitam grandescere, quod, donec erumpat in lucem, mollibus naturae vinculis detinetur; Stat., Silv., I, 6, 4: Saturnus mihi compede exsolu$t a)$. Ora, questa ipotetica Minerva 'legata' sarebbe in realtà il frutto dell'influsso greco, nel dettaglio corinzio, sul mondo etrusco e quindi falisco: infatti, conclude Ferri, "è possibile che Falerii, forse per tramite etrusco, abbia accolto alcuni influssi dal mondo greco, in particolare in ambito dorico, in alcuni usi e nel culto ... Tra questi elementi vi dovette essere anche la consuetudine di incatenare la statua di culto per evitare che abbandonasse la città, usanza diffusa sicuramente presso i Dori e le loro colonie." Ma, non avendo alcuna prova o testimonianza a riguardo, aggiunge che si deve "constatare come nella nostra fonte principale, Ovidio, Minerva non sia concepita né rappresentata in catene: egli non avrebbe certo fatto passare sotto silenzio un particolare così inconsueto", giustificando questo silenzio col fatto che "il poeta di Sulmona la vide più di due secoli dopo ... ovviamente libera da vincoli, necessariamente recisi per far sì che la dea fosse portata a Roma, anche con il grande effetto propagandistico che ciò dovette comportare." (Ferri 2010a, 126). Molto sinteticamente, a netta confutazione di tale proposta, va segnalato che né Ovidio, né altri autori parlano di una Minerva 'incatenata', e non è un dettaglio da poco conto. Va inoltre aggiunto che Saturno, seppur incatenato, non aveva un epiteto che ne sottolineasse la sua condizione, essendo l'atto di legare e slegare un fatto puramente rituale e simbolico. Terza considerazione: il verbo capio non è mai usato col significato di 'legare' ( $c f r$. TLL, III, s. v. capio, 318ss.). Quarta ed ultima osservazione: dal momento che Ovidio cercava quasi ‘disperatamente' delle ipotesi per l'etimologia dell'epiteto Capta, se avesse avuto anche soltanto una seppur confusa tradizione che descrivesse la Minerva del Celio come una divinità 'legata', non si sarebbe potuto di certo esimere dall'esporla tra la interpretazioni proposte nei suoi 


\section{Bibliografia}

F. Altheim, s. v. Minerva, RE 15.2 (1932) 1774-1802.

F. Altheim, Römische religionsgeschichte, I-II, Baden-Baden, 1951-53.

J. Aronen, s. v. Fortuna Viscata / Viscatrix, LTUR 2 (1995) 280-281.

E. Aust, Die Religion der Römer, Münster, 1899.

A. Barchiesi, Il poeta e il principe. Ovidio e il discorso augusteo, Bari, 1994.

V. Basanoff, Evocatio: étude d`un rituel militaire romain, Paris, 1947.

R. Bloch, Recherches sur les religions de l'antiquité classique, Paris, 1980.

M. Bollini, "Minerva Medica Memor", Atti del III convegno di studi veleiati. (Piacenza-Veleia-Parma 1967), Milano - Varese, 1969, 347-358.

$\mathrm{N}$. Boels-Janssen, La vie religieuse des matrones dans la Rome archaique, (CEFR 176), Rome, 1993.

F. Bömer, P. Ovidius Naso. Die Fasten, I-II, Heidelberg, 1957-1958.

J. W. Bouma, "Mater Matuta name and cult: according to ancient sources, approaches of religious research and archaeology", in M. Marskant-Kleibrink (ed.), Images of ancient Latin culture. Caeculus I, Groningen, 1992, 53-75.

A. Brelich, "Vesta", (Albae Vigiliae 7), Zürich, 1949.

P. Bruun, "Evocatio deorum - some notes on the romanization of Etruria", in H. Biezais (ed.), The Myth of the State: based on papers read at the Symposium on the Myth of the State, Stockholm, 1972, 109-120.

C. Buzzetti, s. v. Lucus Facutalis, LTUR 3 (1996a) 193.

C. Buzzetti, s. v. Lucus Esquilinus, LTUR 3 (1996b) 193.

F. Canciani, s. v. Minerva, LIMC 2.1 (1984) 1074-1109.

A. Carandini, La nascita di Roma. Dei, Lari, eroi e uomini all'alba di una civiltà 2, Torino, 1997-2003.

A. Carandini, Remo e Romolo. Dai rioni dei Quiriti alla città dei Romani (775/750 - 700(675 a.C. circa), Torino, 2006.

C. Carlucci, s. v. Minerva Medica, Tempio, LTUR 3 (1996) 255-256.

A. Cassatella, L. Venditelli, "Santuario di Diana sull'Aventino: il problema della localizzazione", Roma. Archeologia nel Centro (LSA 6.2), Roma, 1985, $442-$ 451.

J. Champeaux, Le culte de Fortuna à Rome et dans le monde romain. I. Fortuna dans la religion archaique, (CEFR 64), Rome, 1982.

F. Coarelli, s. v. Argei, Sacraria, LTUR 1 (1993a) 120-125.

F. Coarelli, s. v. Casa Romuli (Cermalus), LTUR 1 (1993b) 241-242.

F. Coarelli, s. v. Minerva Capta, delubra; Minervium, LTUR 2 (1995) 255.

F. Coarelli, s. v. Argei, Sacraria, LTUR 1 (1996) 120-125.

F. Coarelli, "Il tempio di Minerva Capta e la domus di Claudio sul Celio", RPAA 70 (1997-98) 209-218.

F. Coarelli, s. v. Quirinus, Aedes, LTUR 4 (1999a) 185-187.

F. Coarelli, s. v. Salus, Aedes, LTUR 4 (1999b) 229-230.

F. Coarelli, s. v. Semo Sancus in Colle, Aedes, Fanum, Sacellum, Templum, LTUR 4 (1999c) 263-264.

versi. Ipotizzare, poi, che una dea venisse detta e venerata come 'incatenata', ma che nella prassi non lo sia mai stata poiché immediatamente 'liberata' dai Romani al momento della conquista di Falerii, mi sembra a dir poco improbabile. 
A. M. Colini, "Storia e topografia del Celio nell'antichità", MPAA 7 (1944) 39-50.

P. Collart, Varrone. De lingua Latina. Livre V, Paris, 1954.

G. Colonna, s. v. Menerva, LIMC 2.1 (1984) 1050-1074.

G. Colonna, "Acqua Acetosa Laurentina, l'ager Romanus antiquus e i santuari del I miglio", ScAnt 5 (1991) 209-232.

G. Colonna, "Membra disiecta di altorilievi frontonali di IV e III secolo", in G. Maetzke (ed.), La coroplastica templare etrusca fra il IV e il II secolo a.C. Atti del convegno di studi etruschi e italici, Firenze, 1992, 101-126.

S. De Angeli, s. v. Iuppiter Optimus Maximus Capitolinus, Aedes (fasi tardo-repubblicane e di età imperiale), LTUR 3 (1996) 148-153.

A. Degrassi, Inscriptiones Italiae. Vol. XIII - Fasti et Elogia. Fasciculus II - Fasti anni numani et iuliani, Roma, 1963.

M. A. De Lucia Brolli, "Dalla tutela alla ricerca: recenti rinvenimenti dall'area urbana di Falerii", in M. Pandolfini Angeletti (ed.), Archeologia in Etruria meridionale, Civita Castellana, 2003, 65-89.

G. De Sanctis, Storia dei Romani, I-IV, Firenze, 1953.

N. Donati, P. Stefanetti, Dies Natalis. I calendari romani e gli anniversari dei culti, Roma, 2006.

G. Dumézil, "Ner- et Viro- dans les languages italiques", REL 31 (1953) 175-190.

G. Dumézil, La religion romaine archaique, Paris, 1966.

G. Dumézil, Mariages indo-européens, suivi de quinze Questions Romaines, Paris, 1979.

G. Dury-Moyaers, M. Renard, "Aperçu critique de travaux relatifs au culte di Junon", $A N R W 2.17 .1$ (1981) 142-202.

AA. VV., Enea nel Lazio. Archeologia e mito: bimillenario virgiliano. Roma 22 settembre-31 dic. 1981, Campidoglio, Palazzo dei Conservatori, Roma, 1981.

R. Enking, "Minerva Mater", JDAI 59-60 (1944-45) 111-124.

A. Ernout, Morphologie historique du latin, Paris, 1945.

A. Ernout, A. Meillet, Dictionnaire étymologique de la langue latine. Histoire des mots, Paris, 1932.

G. Ferri, "L'evocatio romana. I problemi”, SMSR 30 (2006) 205-244.

G. Ferri, Tutela urbis. Il significato e la concezione della divinità tutelare cittadina nella religione romana, Berlin, 2010a.

G. Ferri, "Una testimonianza epigrafica dell'evocatio? Su un'iscrizione da Isaura Vetus", in S. Antolini, A. Arnaldi, E. Lanzillotta (eds.), Giornata di studi in onore di Lidio Gasperini, Roma, 2010b, 183-194.

G. Ferri, "Due divinità di Falerii Veteres: Giunone Curite e Minerva Capta", MEFRA 123.1 (2011) 145-156 (http://dx.doi.org/10.4000/mefra.489)

G. Fougères, s. v. Minerva, DAGR 3.2 (1969) 1910-1930.

J. G. Frazer, Fastorum libri sex. The Fasti of Ovid. Volume 3: Commentary on Books III and IV, New York, 1973².

J. G. Frazer, Ovid, in six volumes. Fasti, London, $1976^{2}$.

J. Gagé, Apollon romain. Essai sur le culte d'Apollon et le développement du "ritus Graecus" à Rome des origines à Auguste, Paris, 1955.

L. Gatti lo Guzzo, Il deposito votivo dall'Esquilino detto di Minerva Medica, (Studi e materiali di etruscologia e antichità italiche 17), Firenze, 1978.

G. Giannelli, s. v. Iuno Moneta, Aedes, LTUR 3 (1996a) 123-125.

G. Giannelli, s.v. Iuno Lucina, Aedes, LTUR 3 (1996b) 122-123. 
O. Gilbert, Geschichte und Topographie der Stadt Rom im Altertum, I-III, Leipzig, 1883-1890.

J. L. Girard, "Les origines du culte de Minerve", REL 48 (1970) 469-472.

J. L. Girard, "Etude onomastique: le nom de Minerve en Italie", $A E H E$, Ve sect., 80-81 (1971-73) 64-65 (http://dx.doi.org/10.3406/ephe.1971.20724).

J.L. Girard, "La place de Minerve dans la religion romaine au temps du principat", ANRW 2.17.1 (1981) 202-232.

J. L. Girard, "Minerva Capta: entre Rome et Faleries", REL 67 (1989) 163-169.

F. Graf, "Athena and Minerva. Two faces of one goddess?", in A. Deacy, A. Villing (eds.), Athena in the classical world, Leiden, 2001, 127-139.

G. Gustafsson, Evocatio deorum: historical and mythical interpretations of ritualised conquests in the expansion of ancient Rome, Uppsala, 2000.

D. P. Harmon, "The public festivals of Rome", ANRW 2.16.2 (1978) 1440-1468.

K. K. Hersch, The Roman Wedding. Ritual and Meaning in Antiquity, Cambridge, 2010 (http://dx.doi.org/10.1017/CBO9780511762086).

J.A. Hild, s. v. Juno, DAGR 3.1 (1969a) 668-690.

J.A. Hild, s. v. Mater Matuta, DAGR 3.2 (1969b) 1625-1626.

J.A. Hild, s. v. Quinquatrus, DAGR 4.1 (1969c) 802-804.

S. Hinds, "Arma in Ovid's Fasti. Part 1: genre and mannerism", Arethusa 25.1 (1992) 81-112.

Hunziker, s. v. Toga. Prise de la toge virile, DAGR 5 (1969) 347-353.

H. Jordan, C. Hülsen, Topographie der Stadt Rom im Alterthum, I-II, Berlin, 18711907.

T. Köves-Zulauf, "Minerva Capta - Eine gefangene Göttin?”, in Religio Graeco-Romana. Festschrift für Walter Pötscher (GB 5), Horn, 1993, 159-176.

G. La Bua, "Minerva Capta (Ovidio Fasti 3, 809-848)", in G. La Bua (ed.), Vates operose dierum: studi sui Fasti di Ovidio, Pisa, 2010, 51-63.

K. Latte, Römische Religionsgeschichte, München, 1960.

H. Le Bonniec, Ovide. Les Fastes, Paris, 1990.

J. Le Gall, "Evocatio", in Mélanges offerts à Jacques Heurgon. L'Italie préromaine et la Rome républicaine I (CEFR 57), Rome, 1976, 519-524.

A. Ley, s. v. Minerva, in Der Neue Pauly. Enzyklopädie der Antike 8 (2000) 211-216.

E. Marroni, I culti dell'Esquilino, Roma, 2010.

H. G. Martin, Römische Tempelkultbilder. Eine Archäologische Unterschung zur späten Republik, Rome, 1987.

F. H. Massa-Pairault, "De Préneste à Volsinii: Minerve, le fatum et la constitution de la société", PP 42 (1987) 200-235.

M. Menichetti, ...Quoius forma virtutei parisuma fuit... Ciste prenestine e cultura di Roma medio-repubblicana, Roma, 1995.

E. Merli, Arma canant alii: materia epica e narrazione elegiaca nei Fasti di Ovidio, Firenze, 2000.

N. Mezzetti, "Origini del nome e del culto di Minerva", AFLPer 33 (1997-2000) 173-191.

J. F. Miller, "The Fasti and Hellenistic Didactic: Ovid's variant aetiologies", Arethusa 25.1 (1992) 11-31.

J. F. Miller, "Ovid's Liberalia”, in G. Herbert-Brown (ed.), Ovid's Fasti. Historical Readings at its Bimillennium, Oxford, 2002, 199-224 (http://dx.doi.org/10.1093/ acprof:oso/9780198154754.003.0009). 
Montanari, s. v. Evocatio, in G. Filoramo (ed.), Dizionario delle religioni, Torino, 1993, 271.

P. Monteil, Éléments de phonétique et de morphologie du latin, Paris, 1970.

J. M. Moret, "Le feste dei nani", in C. Bocherens (ed.), Nani in festa. Iconografia, religione e politica a Ostia durante il secondo triumvirato, Bari, 2012, 49-108.

A. M. Moretti Sgubini, Le antichità dei Falisci al Museo di Villa Giulia, Roma, 1998.

P. Moscati, "Nuove ricerche su Falerii Veteres", in G. Maetzke (ed.), La civiltà dei Falisci: atti del XV convegno di studi etruschi ed italici. Civita castellana, 28-31 maggio 1987, Firenze, 1990, 162-165.

C. Newlands, "Transgressivs Acts. Ovid's treatment of the Idus of March", CPh 91.4 (1996) 320-338 (http://dx.doi.org/10.1086/367523).

E. Orlin, Temples, Religion and Politics in the Roman Republic, Boston - Leiden, 2002.

R. A. Palmer, The Archaic Community of the Romans, Cambridge, 1970.

D. Palombi, s. v. Penates, Aedes, LTUR 4 (1999) 75-78.

L. Pedroni, "Carvilius, Minerva e Falerii", Latomus 70.1 (2011) 39-50.

E. Peruzzi, "Il voto falisco a Minerva (CIL I² 365)", PP 52 (1997) 61-74.

C. R. Phillips, s. v. Minerva. Cult, in H. Cancik, H. Schneider (eds.), Brill's Encyclopaedia of the Ancient World 8 (2006) 939-943.

G. B. Pighi, La poesia religiosa romana, Bologna, 1958.

G. Pisani Sartorio, s. v. Fortuna et Mater Matuta, Aedes, LTUR 2 (1995) 281-285.

S. B. Platner, T. Ashby, A Topographical Dictionary of Ancient Rome, Roma, 1965 (http://dx.doi.org/10.1017/CBO9781316219706).

J. P. Poe, "The Septimontium and the Subura", TAPhA 108 (1978) 147-154 (http:// dx.doi.org/10.2307/284244).

D. Porte, L'etiologie religieuse dans les 'Fastes'd'Ovide, Paris, 1985.

L. Preller, Römische Mythologie, Berlin, 1858.

L. Preller, H. Jordan, Römische Mythologie, I-III, Berlin, 1881-1883.

A. Prosdocimi, "Le religioni degli Italici", in G. Pugliese Carratelli (ed.) Italia omnium terrarum parens, Milano, 1989, 477-545.

G. Radke, Die Götter Altitaliens, Fontes et Commentationes 3, Münster, 1965.

L. Richardson jr., A New Topographical Dictionary of Ancient Rome, Baltimore, 1992.

H. Rix, "Rapporti onomastici fra il pantheon etrusco e quello romano", in Colonna, C. de Simone, M. Cristofani, J. Heurgon (eds.), Gli Etruschi e Roma. Incontro di studi in onore di Massimo Pallottino. Roma, 11-13 dicembre 1979, Roma, 1981, 104-126.

G. Rohde, Die Kultsatzungen der römischen Pontifices (RGVV 25), Berlin, 1936.

W. H. Roscher, Ausführliches Lexikon der griechischen und römischen Mythologie. I-VII, Leipzig, 1886-

M. Russo, "Punta della Campanella", MonAL 3 (1984-1990) 189-285.

D. Sabbatucci, La religione di Roma antica: dal calendario festivo all'ordine cosmico, Milano, 1988.

L. Sacco, "Devotio", StudRom 52 (2004) 312-352.

E. Saglio, s. v. Argei, DAGR 1.1 (1969) 404-406.

J. Scheid, "Livres sacerdotaux et érudition: 1'exemple des Chapelles des Argées", in C. Batsch, U. Egelhaaf-Gaiser, R. Stepper (eds.), Zwischen Krise und Alltag. Conflit et normalité. Antike Religionem im Mittelmediterraneum. Religions anciennes dans l'espace méditerranéen, Stuttgart, 1999, 161-170. 
J. Scheid, An introduction to Roman Religion, Edinburgh, 2003.

J. Scheid, "Il culto di Minerva in epoca romana e il suo rapporto con la Minerva di Travo", in AA.VV., Minerva Medica in Valtrebbia. Scienze storiche e scienze naturali alleate per la scoperta del luogo di culto, Piacenza, 2008, 85-91.

R. Schillig, s. v. Minerva, in Y. Bonnefoy (ed.), Dictionnaire des Mythologies et des Religions des Sociétés traditionnelles et du Monde Antique 2 (1981) 111-112.

R. Schilling, Ovide. Les Fastes. I, Paris, 2003.

W. Schürmann, Typologie und Bedeutung der stadtrömischen Minerva-Kultbilder (RdA Suppl. 2), Rome, 1985.

H. H. Scullard, Festivals and Cerimonies of the Roman Republic, London, 1981.

G. Stara-Tedde, "I boschi sacri dell'antica Roma”, Bcom 33 (1905) 189-232.

A. Storchi Marino, "Artigiani e rituali religiosi nella Roma arcaica", RAAN 54 (1979) 333-357.

A. Storchi Marino, "Il rituale degli Argei tra annalistica e antiquaria", AIIS 12, 1991/92 (1995) 263-308.

S. Tafaro, Pubes e Viripotentes nella esperienza giuridica romana, Bari, 1988.

G. Tagliamonte, s. v. Capitolium (fino alla prima età repubblicana), LTUR 1 (1996a) 226-231.

G. Tagliamonte, s. v. Iuppiter Optimus Maximus Capitolinus, Aedes, Templum (fino all'A. 83 a.C.), LTUR 3 (1996b) 144-148.

O. Terrosi Zanco, "Varrone LL., V, 74: Divinità Sabine o divinità etrusche?", SCO 10 (1961) 188-208.

M. Torelli, Lavinio e Roma, Roma, 1984.

M. Torelli, "Riti di passaggio maschili di Roma arcaica", MEFRA 102.1 (1990) 93-106 (http://dx.doi.org/10.3406/mefr.1990.1661).

H. Usener, "Italische Mythen", RhM 30 (1875) 182-229.

M. de Vaan, Etymological Dictionary of Latin and the other Italic Languages, Leiden - Boston, 2008.

L. Vendittelli, s. v. Minerva, Aedes (Aventinus), LTUR 3 (1996) 254.

A. Walde, J. B. Hoffmann, Lateinisches etymologisches Wörterbuch, I-II, Heidelberg, 1938.

W. Warde Fowler, Roman Festivals of the Period of the Republic, London, 1899.

G. Wissowa, s. v. Minerva, Roscher 2.2 (1897) 2982-2992.

G. Wissowa, s. v. Capta, RE 3.2 (1899) 1554.

G. Wissowa, Religion und Kultus der Römer, München, 1902.

G. Wissowa, s. v. Evocatio, RE 6.1 (1907) 1152-1153.

G. Wissowa, Gesammelte Abhandlungen zur römisehen Religions-und Stadtgeschichte, München, 1904.

A. Ziolkowski, The temples of mid-republican Rome and their historical and topographical contest, Roma, 1992. 